Trábalho do Departamento de Parasitologia da Faculdade de

Medicina da Universidade de São Paulo

Diretor: Prof. Samuel B. Pessoa

\title{
LISTA DOS PROTOZOÁRIOS HEMOPARASITAS DE AVES DA REGIÃO NEOTRÓPICA
}

\author{
POR \\ Durval T. de Lucena \\ Docente da Fac. de Med. do Recife
}

\section{INTRODUÇÃO}

A idéia da confecção de uma lista dos protozoários hemoparasitas descritos em aves da Região Neotrópica me foi sugerida pelo Prof. Samuel Pessôa quando, em 1938, estive em São Paulo na qualidade de Assistente voluntário de Parasitologia, cadeira de que é catedrático, na Universidade. Tal situação decorreu da benemerência do Dr. Assis Chateaubriand, creador da "Bolsa de Estudos dos Diários Associados».

Naquela época reuni o material bibliográfico necessário, em relação ao que me esforcei para ser o mais completo quanto possivel. Um grande óbice, no entanto, surgiu impossibilitando a redação imediata do trabalho: somente havia sido publicada a primeira parte do "Catálogo das Aves do Brasil», do qual o meu estudo estava dependente pelo entrelaçamento dos assuntos. Graças, porem à excessiva obsequiosidade do Dr. Oliverio M. de Oliveira Pinto, autor daquela magnifica obra, esse escolho foi de pronto removido, prestando-se o erudito ornitologista patrício a esclarecer várias questões concernentes à sinonímia das aves que não constam da primeira parte do seu "Catálogo». Por esse motivo, deixo aquí consignados os meus mais sinceros agradecimentos ao Dr. Oliverio Pinto.

Não posso, de nenhum modo, calar todo o meu entusiasmo e reconhecimento pelo Prof. Samuel Pessôa, a quem devo as lições de mestre e os conselhos de amigo durante o meu aprendizado no seu Laboratório e sempre depois. Por isso, dedico-lhe este trabalho.

Em assunto da natureza do que vou abordar, deveria limitarme à enumeração dos parasitas e seus hospedeiros da maneira mais consentânea; no entanto, si bem que esta seja a conduta geralmente seguida, fui levado a atualizar várias denominações específicas constantes da literatura, dispensando-me de citar os nomes abandonados por demais óbvios. Alguns pontos controvertidos foram maduramente 
pesados antes de serem resolvidos e certas incorreções constantes de livros clássicos foram afastadas, tudo pelo compulsar dos trabalhos originais.

No que respeita aos Toxoplasmas, porem, uma explicação deve ser dada. E' um gênero que, figurando entre os parasitas de natureza duvidosa, tem as suas espécies ainda mais controvertidas, por não apresentarem caracteres próprios que as separem entre si, para tal, algumas vezes, valendo-se os seus autores do velho conceito da hospedagem específica. No que tange às espécies Toxoplasma cuniculi SPLENDORE, 1909 e Toxoplasma columbae Migliano, 1913, encontradas no Pombo, respectivamente, por CARINI e CARINI \& MACIEL, adoto o ponto de vista de Arantes que considera as duas espécies como idênticas ao Toxoplasma gondii (Niccolle \& Manceaux, 1908).

Catalogando os hemoparasitas encontrados em Aves da Região Neotrópica, poderia parecer estranha a referência ao Serinus canarius Linnaeus e Passer domesticus (Linnaeus), pássaros da Europa que para o Brasil foram trazidos em época ainda próxima. O Canário do Império é passarinho de canto e regalo para os criadores afeiçoados; o Pardal infesta cidades e campos dos Estados do Rio de Janeiro, São Paulo e Minas Gerais, tendo sido introduzido no Rio pelo Dr. Francisco Pereira Passos, em (?) 1904.

Dividirei esta lista em três partes. Primeiramente farei a discriminação das aves observadas naturalmente infetadas e os parasitas respetivos; depois enumerarei os hematozoários e, por fim, darei uma lista sinótica das espécies representadas nas aves da nossa região faunística.

Farei constar das duas primeiras listas a indicação bibliográfica em correspondência, tanto quanto possivel, ao local onde foram realizadas as pesquizas.

As aves que foram encontradas parasitadas serão referidas pela ordem alfabética, com os nomes que lhes deram os parasitologistas nos seus escritos originais. Quando alguns deles tiverem sido postos em sinonímia, ulteriormente, serão enunciados entre parênteses seguidos do nome atual. Os nomes atuais serão relacionados independentemente, na mesma ordem, mandando-se ver os sinónimos correspondentes, como foram relatados pelos autores.

Os parasitas serão citados pela ordem sistemática, quanto ao gênero, dentro de cada um desses em primeiro lugar indo colocados os não especificados, enumerando-se todas as aves, com o nome atual, em que foram observados, seguindo-se os demais, especificados, pela ordem alfabética e como acima. Aquí não terei a preocupação do 
nome dado pelos parasitologistas às espécies ornitológicas, pois que esse será encontrado facilmente na lista por ave.

As espécies parasitas serão registadas sistemática e cronológicamente.

Não é esta a primeira vez que semelhante objetivo é visado, entre nós, e aí está para atestar que um roteiro mais ou menos seguro sempre fora desejado para orientar pesquizas aos estudiosos da matéria, o trabalho de CEZAR PINTo - «Protozoarios observados no Brasil». Por outro lado, as varias publicações estrangeiras similares, bem recentes, citadas na bibliografia, são outros exemplos da oportunidade do assunto.

Este trabalho visa, portanto, e, sobretudo, poupar esforço aos que, não tendo bibliografia à mão ou não dispondo de tempo suficiente, desejem inteirar-se do que nele se trata, até à presente data.

Recife, Junho de 1940.

I PARTE

LISTA DAS AVES E DOS HEMOPARASITAS NELAS OBSERVADOS COM INDICAÇÃO BIBLIOGRÁFICA

(AAPTUS CHOPI) $=$ GNORIMOPAR CHOPI CHOPI (Vieillot).

Trypanosoma sp.

Toxoplasma avium Marullaz, 1913. Carini \& Maciel, 1916, S. Paulo, Brasil.

ACCIPITER sp. Vide GAVIÃO.

AGELAIUS ICTEROCEPHALUS Linnaeus.

Haemoproteus danilewskyi Kruse, 1890.

Plimmer, 1913, Mexico.

AIMOPHILA STRIGICEPS DABBENEI (Hellmayr). Vide ZONOTRICHIA STRIGICEPS DABBENEI.

(ANAS MOSCHATA) $=$ CAIRINA MOSCHATA (Linnaeus).

Haemoproteus sp.

Leger, 1918, Guiana Franceza.

APHELOCOMA SORDIDA (Swainson).

Plasmodium praecox Grassi \& Feletti, 1890.

Plimmer, 1914, Mexico.
ARA MACAO (Linnaeus).

Plasmodium praecox Grassi \& Feletti, 1890.

Plimmer, 1912, America Central.

ARAMIDES CAJANEA CAJANEA (Müller).

Plasmodium lutzi Lucena, 1938.

Lucena, 1938d, 1939 e 1939a, S. Paulo, Brasil.

Plasmodium vaughani Novy \& MacNeal, 1904.

Lucena, 1939a, S. Paulo, Brasil.

Toxoplasma sp.

Lucena, 1938, S. Paulo, Brasil.

ARCHIPLANUS ALBIROSTRIS (Vieil. lot). Vide CACICUS CHRYSOPTERUS.

(ARDEA CAERULEA $)=$ FLORIDA CAERULEA (Linnaeus).

Trypanosoma avium minus Danilewsky, 1888.

Cerqueira, 1906, Rio de Janeiro, Brasil. 
(ARDEA CANDIDISSIMA) $=$ LEUCOPHOYX THULA THULA (Molina).

Trypanosoma avium minus Danilewsky, 1888.

Cerqueira, 1906, Rio de Janeiro, Brasil.

(ARDEA CAERULESCENS) $=$ FLORIDA CAERULEA (Linnaeus).

Trypanosoma ardea major Leger, 1918.

Leger, 1918, Guiana Franceza.

(ATTICORA CYANOLEUCUS) $=$ PY. GOCHELIDON CYANOLEUCA CYANOLEUCA (Vieillot).

Plasmodium praecox Grassi \& Feleti, 1890.

Iturbe \& Gonzalez, 1916, Vene. zuela.

Haemogregarina atticorae Aragão, 1911.

Aragão, 1911, Rio de Janeiro, Brasil.

Toxoplasma avium Marullaz, 1913.

Carini \& Maciel, 1916, S. Paulo, Brasil.

(BATARA CINEREA $)=$ BATARA CINEREA CINEREA (Vieillot).

Trypanosoma sp.

Carini \& Botelho, 1914, S. Paulo, Brasil.

BATARA CYNEREA CYNEREA (Vieillot). Vide BATARA CINEREA.

(BATARA MAJOR) = TARABA MAJOR MAJOR (Vieillot).

Haemogregarina travassosi di Primio, 1925.

di Primio, 1925, Rio de Janeiro, Brasil.

(BELONOPTERUS CAYENNENSIS) = BELONOPTERUS CHILENSIS LAMPRONOTUS (Wagler).

Haemogregarina sp.

Neiva \& Penna, 1916, Brasil.

BELONOPTERUS CHILENSIS LAMPRONOTUS (Wagler). Vide BELONOPTERUS CAYNNENSIS.
BOMBYCILLA CEDRORUM (Vieillot).

Leucocytozoon sp.

Beltrán, 1940, Mexico.

(BRACHYSPIZA CAPENSIS) = BRACHISPIZA CAPENSIS MATUTINA (Lichtenstein).

Haemogregarina brachyspizae Aragão, 1911.

Aragão, 1911, Rio de Janeiro, Brasil.

Toxoplasma avium Marullaz, 1913.

Carini \& Maciel, 1916, S. Paulo Brasil.

BRACHISPIZA CAPENSIS MATUTINA (Lichtenstein).

Haemoproteus sp.

Toxoplasma sp.

Lucena, 1938, S. Paulo, Brasil.

BRACHISPIZA CAPENSIS MATUTINA (Lichtenstein).

Vide BRACHYSPIZA CAPENSIS

BRACHYSPIZA PILEATA

ZONOTRICHIA PILEATA

(BRACHYSPIZA PILEATA $)=$ BRACHYSPIZA CAPENSIS MATUTINA (Lichtenstein).

Plasmodium cathemerium Hartman, 1927.

Plasmodium elongatum Huff, 1930. Lucena, 1938a, S. Paulo, Brasil.

Plasmodium nucleophilum Manwell, 1935

Lucena, 1939a, S. Paulo Brasil.

(BUCO MACULATUS STRIATIPECTUS) $=$ NYSTALUS MACULATUS STRIATIPECTUS.

Haemoproteus sp.

Mazza, Gonzalez, Franke \& Gonzalez, 1927, Argentina.

$($ BUTORIDES STRIATA $)=$ BUTORIDES STRIATUS STRIATUS (Linnaeus).

Trypanosoma sp. Leger, 1918, Guiana Franceza. Migone, 1912, Paraguai.

BUTORIDES STRIATUS STRIATUS (Linnaeus). Vide BUTORIDES STRIATA. 
(CACICUS CHRYSOPTERUS) $=$ ARCHIPLANUS ALBIROSTRIS (Vieillot).

Trypanosoma sp. Carini \& Maciel, 1916, S. Paulo, Brasil.

CAIRINA MOSCHATA (Linnaeus). Vide ANAS MOSCHATA.

(CALLISTE CYANOPTERA $)=$ CALOSPIZA CYANOPTERA CYANOPTERA (Ser.).

Haemaproteus danilewskyi Kruse, 1890.

Plimmer, 1912, America do Sul.

Iturbe \& Gonzalez, 1916, Venezuela.

(CALLISTE FESTIVA) $=$ CALOSPIZA CYANOCEPHALA (P. L. S. Müller).

Plimmer, 1912, Brasil.

(CALLISTE MELANONOTA) $=$ CALOSPIZA PERUVIANA (Desmarest).

Haemaproteus danilewskyi Kruse, 1890.

Plimmer, 1912, Brasil.

(CALLISTE THORACICA) = CALOSPIZA DESMARESTI (Vieillot).

Plasmodium praecox Grassi \& Feletti, 1890.

Plimmer, 1912, Brasil.

(CALLISTE TRICOLOR) $=$ CALOSPIZA SELEDON (P. L. S. Müller).

Haemaproteus danilewskyi Kruse, 1890.

Plimmer, 1912, America do Sul.

CALOSPIZA CYANOCEPHALA (P. L. S. Müller). Vide CALLISTE FESTIVA.

CALOSPIZA CYANOPTERA CYANOPTERA (Ser.). Vide CALLISTE CYANOPTERA.

CALOSPIZA DESMARESTI (Vieillot). Vide CALLISTE THORACICA.

CALOSPIZA FASTUOSA (Lesson).

Plasmodium praecox Grassi \& Feletti, 1890.

Scott, 1926, Brasil.
CALOSPIZA PERUVIANA (Desmarest). Vide CALLISTE MELANONOTA.

CALOSPIZA SELEDON (P. L. S. Müller).

Vide CALLISTE TRICOLOR CALOSPIZA TRICOLOR.

(CALOSPIZA TRICOLOR) $=$ CALOSPIZA SELEDON (P. L. S. Müller).

Haemoproteus sp.

Carini \& Maciel, 1916, S. Paulo, Brasil.

$($ CANARIO $)=(?)$ SERINUS CANARIUS Linnaeus.

Toxoplasma avium Marullaz, 1913. Rosenbusch, 1932, Argentina.

(CAPRIMULGUS RUFUS) $=$ SETOCHALCIS RUFA RUFA (Boddaert).

Haemoproteus sp.

Mazza \& Fiora, 1932, Argentina.

CAPSIEMPIS FLAVEOLA (Lichtenstein).

Haemaproteus danilewskyi Kruse, 1890.

Iturbe \& Gonzalez, 1916, Venezuela.

(CARDINALIS PHOENICEUS) $=$ RICHMONDEA PHOENICEA (Bonaparte).

Haemoproteus danilewskyi Kruse, 1890.

Plimmer, 1912, Venezuela.

CARIAMA CRISTATA (Linnaeus).

Haemoproteus sp.

Lutz \& Meyer, 1908, S. Paulo, Brasil.

CARPODACUS MEXICANUS (Müller).

Plasmodium praecox Grassi \& Feletti, 1890.

Plimmer, 1912, Mexico.

Plasmodium sp.

Haemoproteus sp. Beltrán, 1940, Mexico.

CASSICULUS MELANICTERUS (Bonaparte).

Haemoproteus danilewskyi Kruse, 1890.

Plimmer, 1912, Mexico. 
(CATHARISTA ATRATUS) $=$ CORAGYPS ATRATUS FOETENS (Lichtenstein).

Trypanosoma catharistae Mesnil, 1912. Brimont, 1909 e 1912, Guiana Franceza.

Mesnil, 1912, Guiana Franceza.

Haemogregarina pintoi di Primio, 1925.

di Primio, 1925, Rio de Janeiro, Brasil.

$($ CATHARTES AURA $)=$ CATHARTES AURA RUFICOLIS Spix.

Haemoproteus danilewskyi Kruse, 1890.

Darling, 1912, Panamá.

Haemogregarina pintoi di Primio, 1925.

di Primio, 1925, Rio de Janeiro, Brasil.

CATHARTES AURA RUFICOLIS Spix. Vide CATHARTES AURA e CATHARTES BURROVIANUS.

Haemoproteus sp.

Scott, 1912, Brasil.

(CERCHNEIS SPARVERIUS AUSTRALIS) = CERCHNEIS SPARVERIUS EIDOS (Peters).

Trypanosoma langeronianus Mazza \& Fiora, 1932.

Mazza \& Fiora, 1930a e 1932, Argentina.

Haemoproteus sp.

Mazza, Franke \& Alvarado, 1928, Argentina.

CERCHNEIS SPARVERIUS EIDOS

(Peters).

Vide CERCHNEIS SPARVERIUS

AUSTRALIS.

FALCO SPARVERIUS.

CERTHIAXIS CINNAMOMEA RUSSEOLA (Vieillot).

Haemogragarina sp.

Lucena, 1938, S. Paulo, Brasil.

(CHAMAEZA BREVICAUDA) = CHA.

MAEZA BREVICAUDA BREVI-

CAUDA (Vieillot).

Trypanosoma sp.

Carini \& Maciel, 1916, S. Paulo, Brasil.
CHAMAEZA BREVICAUDA BREVICAUDA (Vieillot).

Trypanosoma dabbenei Mazza, Deautier \& Steullet, 1927.

Mazza, Deautier \& Steullet, 1927, Argentina.

CHAMAEZA BREVICAUDA BREVICAUDA (Vieillot). Vide CHAMAEZA BREVICAUDA.

(CHANTHRORNUS JAMAICAI) = ICTERUS JAMAICAII (Gmelin).

Haemoproteus sp.

Aragão, 1916, Rio de Janeiro, Brasil.

(CHLOROPHONIA PRETII) $=$ CHLOROPHONIA PYRROPHRYS (Sclater).

Haemoproteus danilewskyi Kruse, 1890.

Iturbe \& Gonzalez, 1916, Venezuela.

CHLOROPHONIA PYRRHOPHRYS (Sclater). Vide CHLOROPHONIA PRETII.

COCCYZUS MELACORYPHUS Vieillot. Vide COCCYZUS MELANOCORYPHUS.

(COCCYZUS MELANOCORYPHUS) = COCCYZUS MELACORYPHUS Vieillot.

Trypanosoma sp.

Mazza, Franke \& Gonzalez, 1927, Argentina.

(COEREBA CYANEA $)=$ CYANERPES CYANEA CYANEA (Linnaeus).

Haemoproteus danilewskyi Kruse, 1890.

Plimmer, 1912 e 1913, America do Sul.

(COLAPTES CAMPESTRIS) $=$ COLAPTES CAMPESTRIS CAMPES. TRIS (Vieillot).

Plasmodium sp.

Carini \& Maciel, 1916, S. Paulo, Brasil.

COLAPTES CAMPESTRIS CAMPESTRIS (Vieillot). Vide COLAPTES CAMPESTRIS.

COLUMBA sp. Vide POMBO. 
$($ COLUMBA PICAZURO $)=$ COLUMBA PICAZURO PICAZURO Temminck.

Haemoproteus sp. Mazza, Oliva, Schürmann \& Gutdeutsch, 1932, Argentina.

COLUMBA PICAZURO PICAZURO Temminck. Vide COLUMBA PI. CAZURO.

COLUMBA MACULOSA MACULOSA Temminck, Vide NOTIENAS MACULOSA.

(COLUMBA RUFINA) $=$ COLUMBA RUFINA RUFINA Temminck \& Knip.

Haemoproteus sp. Leger, 1918, Guiana Franceza.

COLUMBA RUFINA RUFINA Temminck \& Knip. VIDE COLUMBA RUFINA.

COLUMBA RUFINA SYLVESTRIS Vieillot.

Haemoproteus sp.

Toxoplasma sp. Lucena, 1938, S. Paulo, Brasil.

Haemoproteus columbae Celli \& Sanfelice, 1891.

Lucena, 1939a, S. Paulo, Brasil. COLUMBIGALLINA TALPACOTI

(COLUMBIGALLINA TAPACOTI) = TALPACOTI (Temminck).

Haemoproteus sp.

Aragão, 1916, Rio de Janeiro, Brasil.

COLUMBIGALLINA TALPACOTI TALPACOTI (Temminck).

Haemoproteus sp.

Toxoplasma sp.

Lucena, 1938, S. Paulo, Brasil.

COLUMBIGALLINA TALPACOTI TALPACOTI (Temminck).

Vide COLUMBIGALLINA TALPACOTI.

COLUMBINA TALPACOTI.

(COLUMBINA PICUI) $=$ COLUMBI-

NA PICUI PICUI (Temminck).

Haemoproteus sp.

Mazza \& Fiora, 1932, Argentina. Lucena, 1938, S. Paulo, Brasil.
COLUMBINA PICUI PICUI (Temminck). Vide COLUMBINA PICUI.

(COLUMBINA TALPACOTI) $=\mathrm{CO}$ LUMBIGALLINA TALPACOTI TALPACOTI (Temminck).

Haemoproteus danilewskyi Kruse, 1890.

Iturbe \& Gonzalez, 1916, Venezuela.

CORAGYPS ATRATUS FOETENS (Lichtenstein). Vide CATHARISTA ATRATUS,

CORYPHOSPINGUS CUCULLATUS (Müller).

Haemopruteus sp.

Mazza, Gonzalez, Franke, Gonzalez \& Alvarado, 1927, Argentina. Lucena, 1938, S. Paulo, Brasil.

Toxoplasma sp. Làcena, 1938, S. Paulo, Brasil.

(CRAX ALECTOR) $=$ CRAX NIGRA Linnaeus.

Haemoproteus sp. Brimont, 1912, Guiana Franceza.

CRAX FASCIOLATA Spix. Vide CRAX SCLATERI.

CRAX NIGRA Linnaeus. Vide CRAX ELECTOR.

$($ CRAX SCLATERI $)=$ CRAX FASCIOLATA Spix.

Trypanosoma pedrozi Carini \& Botelho, 1914.

Carini \& Botelho, 1914, S. Paulo, Brasil.

CHROTOPHAGA ANI Linnaeus.

Haemoproteus danilewskyi Kruse, 1890.

Iturbe \& Gonzalez, 1916, Venezuela.

CRYPTURELLUS CINEREUS (Gmelin). Vide CRYPTURUS CINEREUS.

CRYPTURELLUS OBSOLETUS OBSOLETUS (Temminck). Vide CRYP. TURUS OBSOLETUS.

(CRYPTURUS CINEREUS) $=$ CRYPTURELLUS CINEREUS (Gmelin).

Trypanosoma tinami Mesnil, 1912. Mesnil, 1912, Guiana Franceza. Brimont, 1912, Guiana Franceza. 
(CRYPTURUS OBSOLETUS) $=$ CRYPTURELLUS OBSOLETUS OBSOLETUS (Temminck).

Haemopruteus sp.

Brasil.

(CURAEUS ATERRIMUS) $=$ NOTIOPSAR CURAEUS (Molina).

Leucocytozoon sp.

CYANERPES CYANEA CYANEA (Linnaeus). Vide COEREBA CYANEA.

CYANOCOMPSA CYANEA CYANEA (Linnaeus).

Haemogregarina sp.

Lucena, 1938, S. Paulo, Brasil.

(CYANOCORAX CHRYSOPS) $=$ CYANOCORAX CHRYSOPS CHRYSOPS (Vieillot).

Haemoproteus sp.

Lutz \& Meyer, 1908, S. Paulo, Brasil.

Mazza \& Fiora, 1938a, Argentina.

CYANOCORAX CHRYSOPS CHRYSOPS (Vieillot). Vide CYANOCORAX CHRYSOPS.

(CYANOSPIZA LECLANCHERI) = PASSERINA LECLANCHERI L.afresnaye \& Viellot.

Plasmodium praecox Grassi \& Feletti, 1890.

Plimmer, 1912, Mexico.

(DACNIS CAYANA $)=$ DACNIS CAYANA CAYANA (Linnaeus).

Plasmodium praecox Grassi \& Feletti, 1890.

Plimmer, 1912, America do Sul.

Haemoproteus sp.

Pessôa, 1935, S. Paulo, Brasil.

DACNIS CAYANA CAYANA (Linnaeus). Vide DACNIS CAYNA.

(ELAENEA ALBICEPS) = ELAENIA ALBICEPS ALBICEPS (Lafresnaye 8: d'Orbigny).

Toxoplasma avium Marullaz, 1913.

Carini \& Maciel, 1916, S. Paulo, Brasil.

ELAENIA ALBICEPS ALBICEPS (Lafresnaye \& d'Orbigny). Vide ELAENEA ALBICEPS.
(ERYTHROCNEMA UNICINCTA) = PARABUTEO UNICINCTUS UNICINCTUS (Temminck).

Haemoproteus danilewskyi Kruse, 1890.

Iturbe \& Gonzalez, 1916, Venezuela.

EUETHIA CANORA (Gmelin). Vide PHONIPARA CANORA.

(EUPHONIA VIOLACEA) = TANAGRA VIOLACEA VIOLACEA Linnaeus.

Haemoproteus danilewskyi Kruse, 1890.

Plasmodium praecox Grassi \& Feletti, 1890.

Iturbe \& Gonzalez, 1916, Venezuela.

EUXENURA GALEATA (Molina). Vide EUXENURA MAGUARI.

(EUXENURA MAGUARI) = EUXENURA GALEATA (Molina).

Haemoproteus sp. Carini \& Maciel, 1916, S. Paulo, Brasil.

(FALCO SPARVERIUS) $=\mathrm{CERCH}-$ NEIS SPARVERIUS EIDOS (Peters).

Haemoproteus sp. Leger, 1918, Guiana Franceza.

FLORIDA CAERULEA (Linnaeus). Vide ARDEA CAERULEA ARDEA CAERULESCENS.

GALLUS GALLUS DOMESTICUS Linnaeus.

Toxoplasma sp. Lucena, 1938, S. Paulo, Brasil.

$($ GAVIÃO) $=$ ACCIPITER sp.

Haemoproteus sp. Lutz \& Meyer, 1908, S. Paulo, Brasil.

GERANOSPIZA CAERULESCENS CAERULESCENS (Vieillot). Vide GERANOSPIZA GRACILIS.

(GERANOSPIZA GRACILIS) = GERANOSPIZA CAERULESCENS CAERULESCENS (Vieillot).

Haemoproteus danilewskyi Kruse, 1890.

Iturbe \& Gonzalez, 1916, Venezuela. 
GNORIMOPAR CHOPI CHOPI (Vieillot). Vide AAPTUS CHOPI.

GRALLARIA VARIA IMPERATOR Lafresnaye.

Trypanosoma sp.

Carini \& Botelho, 1914, S. Paulo, Brasil.

$($ GYPAGUS PAPA $)=$ SARCORAMPHUS PAPA (Linnaeus).

Toxoplasma avium Marullaz, 1913. Carini \& Maciel, 1916, S. Paulo, Brasil.

(HADROSTOMUS RUFUS) $=$ PLATYPSARIS RUFUS RUFUS (Vieillot).

Haemoproteus sp.

Carini \& Maciel, 1916, S. Paulo, Brasil.

Pessôa, 1935, S. Paulo, Brasil.

HARPIA HARPYJA (Linnaeus). Vide THRASAETUS HARPYJA.

(HETEROSPIZIAS MERIDIONALIS) $=$ HETEROSPIZIAS MERIDIONALIS MERIDIONALIS (Latham).

Trypanosoma mesnili guyanense Mesnil, 1912.

Mesnil, 1912, Guiana Franceza. Brimont, 1912, Guiana Franceza.

HETEROSPIZIA MERIDIONALIS MERIDIONALIS (Latham). Vide HETEROSPIZIAS MERIDIONALIS.

ICTERUS JAMACAII (Gmelin). Vide CHANTORNUS JAMAICAI ICTERUS JAMAICAI

(ICTERUS JAMAICAI) $=$ ICTERUS JAMACAII (Gmelin).

Plasmodium praecox Grassi \& Feletti, 1890.

Plimmer, 1912, Brasil.

Trypanosoma avium Danilewsky, 1885. Plimmer, 1914, Brasil.

(LEISTES GUIANENSIS) = LEISTES MILITARIS MILITARIS (Linnaeus).

Trypanosoma avium Danilewsky, 1885. Plimmer, 1912, Guiana Ingleza.

LEISTES MILITARIS MILITARIS (Linnaeus). Vide LEISTES GUIANENSIS.
(LEPIDOCOLAPTES ANGUSTIROSTRIS) $=$ LEPIDOCOLAPTES ANGUSTIROSTRIS ANGUSTIROSTRIS (Vieillot).

Haemoproteus sp.

Mazza, Gonzalez, Franke, Gonzalez \& Alvarado, 1927, Argentina.

LEPIDOCOLAPTES ANGUSTIROSTRIS ANGUSTIROSTRIS (Vieillot). Vide LEPIDOCOLAPTES ANGUSTIROSTRIS.

LEUCOPHOYX THULA THULA (Molina). Vide ARDEA CANDIDISSIMA.

LEUCOPTERNIS ALBICOLLIS ALBICOLLIS (Latham). Vide URUBITINGA ALBICOLLIS.

(MACHETORNES RIXOSA) = MACHETORNES RIXOSA RIXOSA (Vieillot).

Haemoproteus sp.

Mazza \& Fiora, 1932, Argentina.

MACHETORNES RIXOSA RIXOSA (Vieillot). Vide MACHETORNES RIXOSA.

$($ MACUCO $)=$ TINAMUS $\mathrm{sp}$.

Haemoproteus sp.

Lutz \& Meyer, 1908, S. Paulo, Brasil.

(MILVAGO CHIMACHIMA) $=$ MILVAGO CHIMACHIMA CHIMACHIMA (Vieillot).

Haemoproteus danilewskyi Kruse, 1890.

Iturbe \& Gonzalez, 1916, Venezuela.

MILVAGO CHIMACHIMA CHIMACHIMA (Vieillot). Vide MILVAGO CHIMACHIMA.

MIMUS SATURINUS MODULATOR (Gould).

Plasmodium cathemerium Hartman, 1927.

Lucena, 1939a, S. Paulo, Brasil.

MOLOTHRUS sp.

Plasmodium sp.

Beltrán, 1940, Mexico. 
(MOLOTHRUS BADIUS) $=$ MOLOTHRUS BADIUS BADIUS (Vieillot).

Haemoproteus sp.

Mazza, Gonzalez, Franke, Gonzalez \& Alvarado, 1927, Argentina.

Trypanosoma sp.

Mazza, Franke \& Gonzalez, 1927, Argentina.

MOLOTHRUS BADIUS BADIUS (Vieillot). Vide MOLOTHRUS BADIUS

MOLOTHRUS BONARIENSIS BONARIENSIS (Gmelin).

Plasmodium cathemerium Hartman, Lucena, 1938c, S. Paulo, Brasil.

Haemoproteus sp.

Toxoplasma sp.

Lucena, 1938, S. Paulo, Brasil.

Haemogregarina sp.

Corrêa, 1928, S. Paulo, Brasil.

MUSCIVORA TYRANNUS (Linnaeus). Haemoproteus sp.

Carini \& Maciel, 1916, S. Paulo. Brasil.

MYCTERIA AMERICANA (Linnaeus).

Haemoproteus sp.

Lutz \& Meyer, 1908, S. Paulo, Brasil.

MYCTERIA AMERICANA Linnaeus. Vide TANTALUS AMERICANUS.

MYIOBORUS BRUNNEICEPS d'Orbigny.

Haemoproteus sp.

Mazza \& Fiora, 1932, Argentina.

MYIOZETETES SIMILIS SIMILIS

(Spix). Vide MYIOZETETES TEXENSIS.

(MYIOZETETES TEXENSIS) $=$ MYOZETETES SIMILIS SIMILIS (Spix).

Haemoproteus danilewskyi Kruse, 1890.

Iturbe \& Gonzalez, 1916, Venezuela.

(NOTIENAS MACULOSA) = CO-

LUMBA MACULOSA MACULOSA

Temminck.

Haemoproteus sp.

Mazza, Gonzalez, Franke, Gonzalez \& Alvarado, 1927, Argentina. NOTIOPSAR CURAEUS (Molina). Vide CURAEUS ATERRIMUS.
(NYCTANASSA VIOLACEA $)=$ NYCTANASSA VIOLACEA CAYENNENSIS (Gmelin).

Trypanosoma ardea Leger, 1918. Leger, 1918, Guiana Franceza.

Haemoproteus sp. ou Plasmodium praecox Grassi \& Feletti, 1890.

Scott, 1926, America do Sul.

NYCTANASSA VIOLACEA CAYENNENSIS (Gmelin). Vide NYCTANASSA VIOLACEA.

NYCTICORAX sp.

Haemoproteus sp.

Lutz \& Meyer, 1908, S. Paulo Brasil.

$($ NYCTICORAX GARDENIA $)=$ NYCTICORAX NYCTICORAX HOACTLI (Gmelin).

Trypanosoma avium minus Danilewski, 1888.

Cerqueira, 1906, Rio de Janeiro, Brasil.

Aragão, 1906, Rio de Janeiro, Brasil, «in» Cerqueira, 1906, Brasil.

NYCTICORAX NYCTICORAX HOACTLI (Gmelin). Vide NYCTICORAX GARDENIA.

NYSTALUS MACULATUS STRIATIPECTUS. Vide BUCCO MACULATUS STRIATIPECTUS.

(ODONTOPHORUS CAPUEIRA) = ODONTOPHORUS CAPUEIRA CAPUEIRA (Spix).

Haemoproteus sp.

Lutz \& Meyer, 1908, S. Paulo, Brasil.

ODONTOPHORUS CAPUEIRA CAPUEIRA (Spix). Vide ODONTOPHORUS CAPUEIRA.

(ORTALIS CANICOLLIS) $=$ ORTALIS CANICOLLIS PANTANALENSIS Cherrie \& Reichenberger.

Haemoproteus sp.

Mazza, Deautier \& Steullet, 1930, Argentina.

Mazza \& Fiora, 1932, Argentina. Romaña, 1932, Argentina, «in» Mazza \& Fiora, 1932, Argentina. 
ORTALIS CANICOLLIS PANTANALENSIS Cherrie \& Reichenberger. Vide ORTALIS CANICOLLIS.

(OTUS CHOLIBA) $=$ OTUS CHOLIBA CHOLIBA (Vieillot).

Haemoproteus sp.

Mazza, Franke \& Alvarado, 1928, Argentina.

OTUS CHOLIBA CHOLIBA (Vieillot). Vide OTUS CHOLIBA e SCOPS BRASILIANUS.

OTUS CHOLIBA CRUCIGERUS (Spix). Vide SCOPS BRASILIANUS.

(OTUS CLAMATOR) $=$ RHYNOPTYNX CLAMATOR CLAMATOR (Vieillot).

Haemoproteus sp.

di Primio, 1925, Rio de Janeiro, Brasil.

PACHYRHAMPHUS POLYCHOPTERUS POLYCHOPTERUS (Vieillot). Vide PACHYRHAMPHUS POLYCHOPTERUS.

(PACHYRHAMPHUS POLYCHOPTERUS) $=$ PACHYRHAMPHUS POLYCHOPTERUS POLYCHOPTERUS (Vieillot).

(Vieillot).

Haemoproteus sp.

Carini \& Maciel, 1916. S. PAULO, Brasil.

PARABUTEO UNICINCTUS UNICINCTUS (Temminck). Vide ERYTHROCNEMA UNINICINCTA.

(PAROARIA CAPITATA $)=$ PAROARIA CAPITATA CAPITATA (Lafresnaye \& d'Orbigny).

Haemogregarina aragãoi de Primio, 1925.

di Primio, 1925, Rio de Janeiro, Brasil.

PAROARIA CAPITATA CAPITATA (Lafresnaye \& d'Orbigny). Vide PAROARIA CAPITATA.

PAROARIA CORONATA (Müller). Vide PAROARIA CRISTATA.

PAROARIA DOMINICANA (Linnaeus). Vide PAROARIA LARVATA.
(PAROARIA CRISTATA) = PAROARIA CORONATA (Müller).

Trypanosoma balfouri Mazza, Oliva, Schürmann \& Gutdeutsch, 1932. Mazza, Oliva, Schürmann \& Gutdeutsch, 1932, Argentina.

(PAROARIA LARVATA $)=$ PAROARIA DOMINICANA (Linnaeus).

Haemogregarina paroariae Aragão, 1911.

PASSER DOMESTICUS (Linnaeus). Haemoproteus sp.

Petrochi \& Zuccarini, 1925, Argentina.

Vogelsang, «in» Cordero, 1928, Argentina.

Plasmodium praecoox Grassi \& Feletti, 1890.

Petrochi \& Zuccarini, 1925, Argentina.

Plasmodium cathemerium Hartman, 1927.

Lucena, 1938a, S. Paulo, Brasil.

Plasmodium nucleophilum Huff, 1930. Lucena, 1938b, S. Paulo, Brasil.

PASSERINA LECLANCHERI Lafresnaye $\&$ Vieillot. Vide CYANOSPIZA LECLANCHERI.

(PATO DO MATO).

Haemoproteus sp.

Lutz \& Meyer, 1908, S. Paulo, Brasil.

$($ PENELOPE OBSCURA $)=$ PENELO PE OBSCURA BRONZINA Hellmayr.

Haemoproteus sp.

Lutz, \& Meyer, 1908, S. Paulo, Brasil.

PENELOPE OBSCURA BRONZINA Hellmayr. Vide PENEPOLE OBSCURA.

PENELOPE PILEATA Wagler.

Haemoproteus sp. Scott, 1926, America do Sul.

$($ PENELOPE SUPERCILIARIS $)=$ PENELOPE SUPERCILIARIS JACUPEMBA Spix.

Haemoproteus sp. Carini \& Maciel, 1916, S. Paulo, Brasil. 
PENELOPE SUPERCILIARIS JACUPEMBA Spix. Vide PENELOPE SUPERCILIARIS.

PEZITES DEFILIPPII (Bonaparte). Vide STURNELLA DEFILIPPII. (PHEUCTICUS AUREIVENTRIS) = PHEUCTICUS AUREO-VENTRIS AUREO-VENTRIS (Lafresnaye \& d'Orbigny).

Haemoproteus mazzai Parodi \& Niño, 1926.

Parodi \& Niño, 1926 e 1927, Argentina.

PHEUCTICUS AUREO-VENTRIS AUREO-VENTRIS (Lafresnaye \& d'Orbigny). Vide PHEUCTICUS AUREIVENTRIS.

$($ PHONIPARA CANORA $)=$ EUETHIA CANORA (Gmelin).

Plasmodium praecox Grassi \& $\mathrm{Fe}$ letti, 1890.

Plimmer, 1912, Cuba.

PIAYA sp.

Haemoproteus sp.

Neiva \& Penna, 1916, Brasil.

PIAYA CAYANA MACROURA Gambel.

Trypanosoma sp.

Carini \& Botelho, 1914, S. Paulo Brasil.

PIONUS MENSTRUUS (Linnaeus).

Haemoproteus sp.

Scott, 1926, America do Sul.

(PIRANGA AZARAE) $=$ PIRANGA FLAVA FLAVA (Vieillot).

Haemoproteus sp.

Mazza \& Fiora, 1932, Argentina.

(PIRANGA FLAVA) $=$ PIRANGA FLAVA FLAVA (Vieillot).

Leucocytozoon sp.

Trypanosoma sp.

Haemoproteus sp.

Mazza \& Fiora, 1930, Argentina.

PIRANGA FLAVA FLAVA (Vieillot). Vide PIRANGA AZARAE e PIRANGA FLAVA.
(PITANGUS SULPHURATUS) $=$ PITANGUS SULPHURATUS MAXIMILIANI (Cabanis \& Heine).

Haemoproteus danilewskyi Kruse, 1890.

Plimmer, 1914, América do Sul.

Toxoplasma avium Marullaz, 1913.

Carini \& Maciel, 1916, S. Paulo,

PITANGUS SULPHURATUS BOLIVIANUS (Lafresnaye).

Leucocitozoon sp.

Mazza \& Fiora, 1930, Argentina.

Haemoproteus sp.

Mazza, Steullet \& Deautier, 1932, Argentina.

PITANGUS SULPHURATUS MAXIMILIANI (Cabanis \& Heine).

Trypanosoma sp.

Carini \& Botelho, 1914, S. Paulo, Brassil.

Haemoproteus danilewskyi Kruse, 1890.

Iturbe \& Gonzalez, 1916, Venezuela.

PITANGUS SULPHURATUS MAXIMILIANI (Cabanis \& Heine). Vide PITANGUS SULPHURATUS.

(PLANESTICUS ALBIVENTER) = TURDUS LEUCOMELAS ALBIVENTER Spix.

Haemoproteus danilewskyi Kruse, 1890.

Iturbe \& Gonzalez, 1916, Venezuela.

(PLANESTICUS RUFIVENTRIS) $=$ TURDUS CHIGUANCO ANTHRACINUS (Burmeister).

Plasmodium vaughani Novy \& MacNeal, 1904.

Mazza \& Fiora, 1930, Argentina.

(PLANESTICUS RUFIVENTRIS) = TURDUS RUFIVENTRIS RUFIVENTRIS Vieillot.

Trypanosoma sp.

Pessôa, 1935, S. Paulo, Brasil.

PLATIPSARIS RUFUS RUFUS (Vieillot). Vide HADROSTOMUS RUFUS.

POLYBORUS PLANCUS BRASILIENSIS. (Gmelin). Vide POLYBORUS THARUS. 
$($ POLYBORUS THARUS $)=$ POLYBORUS PLANCUS BRASILIENSIS (Gmelin).

Haemoproteus sp.

Carini \& Maciel, 1916, S. Paulo, Brasil.

$(\mathrm{POMBO})=$ COLUMBA sp.

Haemoproteus sp.

Aragão, 1907, 1907a, 1908, Rio de Janeiro, Brasil.

Leger, 1918, Guiana Franceza.

Haemoproteus columbae Celli \& Sanfelice, 1891.

Carini, 1912, S. Paulo, Brasil.

Aragão, 1916, Rio de Janeiro, Brasil.

di Primio, 1934, Rio Grande do Sul, Brasil.

Toxoplasma gondii (Nicolle \& Manceaux, 1908).

Arantes, 1914, Rio de Janeiro, Brasil.

Carini, 1911, S. Paulo, Brasil.

Carini \& Maciel, 1916, S. Paulo, Brasil,

POOSPIZA THORACICA (Nordmann).

Haemogregarina pessoai Corrêa, 1928. Corrêa, 1928, S. Paulo, Brasil.

PSITTACARA ACUTICAUDATA ACUTICAUDATA (Vieillot). Vide THECTOCERCUS ACUTICAUDATUS.

PYGOCHELIDON CYANOLEUCA CYANOLEUCA (Vieillot). Vide ATTICORA CYANOLEUCUS.

RAMPHASTUS TOCO Müller.

Haemoproteus danilewskyi Kruse, 1890.

Iturbe \&: Gonzalez, 1916, Venezuela.

(RAMPHOCOELUS BRASILIUS) = RAMPHOCELUS BRESILIUS BRESILIUS (Linnaeus).

Haemogregarina rhamphocoeli Aragão, 1911.

Aragão, 1911, Rio de Janeiro, Brasil.

Plasmodium praecox Grassi \& Feletti, 1890.

Scott, 1926, Brasil.
RHAMPHOCELUS BRESILIUS BRESILIUS (Linnaeus). Vide RHAMPHOCOELUS BRASILIUS.

(RHEA AMERICANA) = RHEA AMERICANA INTERMEDIA Rothschild \& Chubb.

Plasmodium sp.

Carini \& Maciel, 1916, S. Paulo, Brasil.

RHEA AMERICANA INTERMEDIA Rothschild \& Chubb. Vide RHEA AMERICANA.

RHYNOPTYNX CLAMATOR CLAMATOR (Vieillot). Vide OTUS CLAMATOR.

RICHMONDEA PHOENICEA (Bonaparte). Vide CARDINALIS PHOENICEUS.

$($ RUPORNIS LEUCORRHEA $)=$ RUPORNIS LEOCORRHOUS (Gnoy \&. Gaimard).

Haemoproteus danilewskyi Kruse, 1890.

Iturbe \& Gonzalez, 1916, Venezuela.

RUPORNIS LEUCORRHOUS (Gnoy \& Gaimard). Vide RUPORNIS LEUCORRHEA.

SALTATOR SIMILIS Lafresnaye \& d'Orbigny.

Haemogregarina sp.

Lucena, 1938, S. Paulo, Brasil.

(SARACURA)

Plasmodium sp.

Lutz \& Meyer, 1908, S. Paulo, Brasil.

SARCORAMPHUS PAPA (Linnaeus).

SCARDAFELLA SQUAMMATA SQUAMMATA (Lesson), Vide SCARDAFELLA SQUAMOSA.

(SCARDAFELLA SQUAMOSA) = SCARDAFELLA SQUAMMATA SQUAMMATA (Lesson).

Plasmodium praecox Grassi \& Feletti, 1890.

Plimmer, 1912, América do Sul.

Haemoproteus sp.

Neiva \& Penna, 1916, Brasil. 
(SCHISTOCHLAMYS CAPISTRATUS) $=$ SCHISTOCHLAMYS RUFICAPILLUS CAPISTRATUS (NeUwied).

Trypanosoma schistochlamydis Splendore, 1910.

Splendore, 1910, S. Paulo, Brasil.

SCHISTOCHLAMYS RUFICAPILLUS CAPISTRATUS (Neuwied). Vide SCHISTOCHLAMYS CAPISTRATUS.

(SCOPS BRASILIANUS) $=$ OTUS CHOLIBA CRUCIGERUS (Spix).

Haemoproteus sp.

Leger, 1918, Guiana Franceza.

(SCOPS BRASILIANUS) $=$ OTUS CHOLIBA CHOLIBA (Vieillot).

Trypanosoma sp.

Haemoproteus sp.

Leucocytozoon sp.

Lutz \& Meyer, 1908, S. Paulo, Brasil.

Leucocytozoon lutzi Carini, 1920.

Haemoproteus sp.

Carini, 1920, S. Paulo, Brasil.

SCOTOPELIA BOUVIERI (Scharpe).

Haemoprotens danilewskyi Kruse, 1890.

Plimmer, 1912, America do Sul.

SERINUS CANARIUS Linnaeus.

Plasmodium praecox Grassi \& $\mathrm{Fe}$ letti, 1890. (?).

Reis \& Nobrega, 1936, S. Paulo, Brasil.

SERINUS CANARIUS Linnaeus, Vide CANARIO.

SETOCHALCIS RUFA RUFA (Boddáert). Vide CAPRIMULGUS RUFUS.

(SICALIS FLAVEOLA) $=$ SICALIS FLAVEOLA FAVA (Müller).

Toxoplasma sicalidis (Aragão, 1911). Nöller, 1920, «in» Wenyon, 1926.

Haemoproteus danilewskyi Kruse, 1890.

Iturbe \& Gonzalez, 1916, Venezuela.

SICALIS FLAVEOLA FLAVA (Müller). Vide SICALIS FLAVEOLA.
SICALIS LUTEOLA LUTEIVENTRIS (Meyer). SYCALIS ARVENSIS.

(SPERMOPHILUS sp.) = SPOROPHILA sp.

Haemoproteus sp.

Lutz \& Meyer, 1908, S. Paulo, Brasil.

SPOROPHILA sp. Vide SPERMOPHILUS sp.

SPOROPHILA ALBOGULARIS (Spix).

Haemogregarina sporophilae Aragão, 1911.

Aragão, 1911, Rio de Janeiro, Brasil.

Toxoplasma sporophilae Nöller, 1920. Nöller, 1920 «in» Wenion, 1926.

Toxoplasma avium Marullaz, 1913. Carini \& Maciel, 1916, S. Paulo, Brasil.

SPOROPHILA CAERULESCENS Vieillot.

Toxoplasma sp.

Lucena, 1938, S. Paulo, Brasil.

STEPHANOPHORUS DIADEMATUS (Temminck). Vide STEPHANOPHORUS LEANCOCEPHALUS.

(STEPHANOPHORUS LEANCOCEPHALUS) $=$ STEPHANOCEPHORUS DIADEMATUS (Temminck).

Haemogregarina paulasousai Corrêa, 1928.

Corrêa, 1928, S. Paulo, Brasil.

$($ STHIX FLAMMEA $)=$ TYTO ALBA TUIDARA (Gray).

Haemoproteus sp.

Lutz \& Meyer, 1908, S. Paulo, Brasil.

(STURNELLA DEFILIPPI) $=$ PEZITES DEFILIPPII (Bonaparte).

Haemoprotcus danilewskyi Kruse, 1890.

Plimmer, 1913, Chile.

(SYCALIS ARVENSIS) = SICALIS LUTEOLA LUTEIVENTRIS (Meyer).

Leucocytozoon sp.

Scott, 1926, Chile. 
SYCALIS COLUMBIANA Cabanis.

Plasmodium praecox Grassi \& Feletti, 1890.

Iturbe \& Gonzalez, 1916, Venezuela.

SYNALLAXIS RUFICAPILLA (Vieillot).

Plasmodium sp.

Carini \& Maciel, 1916, S. Paulo, Brasil.

(TACHYPHONUS CORONATUS) $=$ TACHYPHONUS RUFUS CORONATUS (Vieillot).

Haemogregarina sp.

Lucena, 1938, S. Paulo, Brasil.

(TACHYPHONUS ORNATA) = THRAUPIS ORNATA (Sparrman).

Trypanosoma sp.

Cerqueira, 1906, Rio de Janeiro Brasil.

TACHYPHONUS RUFUS CORONATUS (Vieillot).

Vide TACHYPHONUS CORONATUS

TECHYPHOCEUS CORONATUS.

$($ TAENIOPTERA NEUGETA $)=$ XOLMIS CINEREA Vieillot.

Haemoproteus sp.

Carini \& Maciel, 1916, S. Paulo, Brasil.

TANAGRA ATRICAPILLA Gmelin. Vide TRINGA ATRICAPILLA.

TANAGRA CYANOPTERA Vieillot.

Haemoproteus sp.

Pessôa, 1935, S. Paulo, Brasil.

(TANAGRA EPISCOPUS) $=$ THRAPIS EPISCOPUS Linnaeus.

Haemoproteus danilewskyi Kruse 1890.

Plimmer, 1912, América do Sul.

$($ TANAGRA PALMARUM $)=$ THRAUPIS PALMARUM PALMARUM (Neuwied).

Haemoproteus danilewskyi Kruse 1890.

Plimmer, 1912, América do Sul.

Haemogreagrina tanagrae Aragão, 1911.

Aragão, 1911, Rio de Janeiro, Brasil.

Toxoplasma avium Marullaz, 1913.

Carini \& Maciel, 1916, S. Paulo, Brasil.
(TANAGRA PALMARUM) = THRAUPIS PALMARUM MELANOPTERA Sclater.

Haemoproteus danilewskyi Kruse, 1890. Iturbe \& Gonzalez, 1916, Venezuela.

(TANAGRA SAYACA) $=$ THRAUPIS SAYACA SAYACA (Linnaeus).

Haemoproteus sp. Carini \& Maciel, 1916, S. Paulo, Brasil.

Toxoplasma sp.

Haemogregarina sp. Pessôa, 1929, S. Paulo, Brasil.

TANAGRA VIOLACEA VIOLACEA Linnaeus. Vide EUPHONIA VIOLACEA.

(TANTALUS AMERICANUS) $=$ MYCTERIA AMERICANA Linnaeus.

Trypanosoma sp. Migone, 1916, Paraguai.

TARABA MAJOR MAJOR (Vieillot). Vide BATARA MAJOR.

(TECHYPHOCEUS CORONATUS) =

TACHYPHONUS RUFUS CORONATUS (Vieillot).

Toxoplasma $\mathrm{sp}$. Pessôa, 1929, S. Paulo, Brasil.

(THAMNOPHILUS RUFICAPILLUS) $=$ THAMNOPHILUS RUFICAPILLUS RUFICAPILLUS Vieillot.

Plasmodium vaughani Novy \& MacNeal, $19 \overline{0} 4$.

Lucena, 1939a, S. Paulo, Brasil.

THAMNOPHILUS RUFICAPILLUS

RUFICAPILLUS Vieillot. Vide THAMNOPHILUS RUFICAPILLUS.

(THECTOCERCUS ACUTICAUDATUS) $=$ PSITTACARA ACUTICAUDATA ACUTICAUDATA (Vieillot).

Trypanosoma balfouri Mazza, Oliva, Schürmann \& Gutdeutsch, 1932.

Mazza, Oliva, Schürmann \& Gutdeutsch, 1932, Argentina.

(THERISTICUS CAUDATUS) $=$ THERISTICUS CAUDATUS CAUDA. TUS (Boddaert).

Trypanosoma sp. Migone, 1916, Paraguai. 
THERISTICUS CAUDATUS CAUDATUS (Boddaert). Vide THERISTICUS CAUDATUS.

(THRASAETUS HARPYJA) $=$ HARPIA HARPYJA (Linnaeus).

Trypanosoma sp.

Iturbe \& Gonzalez, 1916, Venezuela.

THRAUPIS sp.

Haemoproteus sp.

Lucena, 1938, S. Paulo, Brasil.

THRAUPIS BONARIENSIS (Gmelin).

Haemoproteus sp.

Mazza, Gonzalez, Franke, Gonzalez \& Alvarado, 1927, Argentina.

(THRAUPIS CANA) $=$ THRAUPIS EPISCOPUS CANA (Ser.).

Haemoproteus danilewskyi Kruse 1890.

Iturbe \& Gonzalez, 1916, Venezuela.

THRAUPIS EPISCOPUS Linnaeus, Vide TANAGRA EPISCOPUS.

THRAUPIS EPISCOPUS CANA (Ser.). Vide THRAUPIS CANA.

THRAUPIS ORNATA (Sparrmann). Vide TACHYPHONUS ORNATA.

THRAUPIS PALMARUM MELANOPTERA Sclater. Vide TANAGRA PALMARUM.

THRAUPIS PALMARUM PALMARUM (Neuwied). Vide TANAGRA PALMARUM.

THRAUPIS SAYACA SAYACA (Linnaeus). Vide TANAGRA SAYACA.

TINAMUS sp. Vide MACUCO.

TINAMUS MAJOR MAJOR (Gmelin). Vide TINAMUS SUBCRISTATUS.

(TINAMUS SUBCRISTATUS) $=$ TINAMUS MAJOR MAJOR (Gmelin).

Trypanosoma tinami Mesnil, 1912.

Brimont 1912, Guiana Franceza.

Mesnil, 1912, Guiana Franceza.

(TITYRA BRASILIENSIS) $=$ TITYRA CAYANA BRASILIENSIS (Swainson).

Trypanosoma sp.

Pessôa, 1935, S. Paulo, Brasil.

TITYRA CAYANA BRASILIENSIS (Swaison). Vide TITYRA BRASILIENSIS.
(TRINGA ATRICAPILLA) Gmelin.

Haemoproteus sp.

Leger, 1918, Guiana Franceza.

(TROGON ATRICOLLIS) $=$ TROGONURUS RUFUS RUFUS (Gamelin).

Haemoproteus danilewskyi Kruse, 1890. Iturbe \& Gonzalez, 1916, Venezuela.

TROGONURUS RUFUS RUFUS (Gmelin). Vide TROGON ATRICOLLIS.

TURDUS CHIGUANCO ANTHRACINUS (Burmeister). Vide PLANESTICUS ANTHRACINUS.

(TURDUS FUMIGATUS) $=$ TURDUS FUMIGATUS FUMIGATUS Lichtenstein.

Haemoproteus danitewskyi Kruse 1890.

Iturbe \& Gonzalez, 1916, Venezuela. TURDUS FUMIGATUS FUMIGATUS Lichtenstein. Vide TURDUS FUMIGATUS.

TURDUS LEOCOMELAS ALBIVENTER Spix. Vide PLANESTICUS ALBIVENTER.

TURDUS LEUCOMELAS LEUCOMELAS Vieillot.

Plasmodium vaughani Novy \& MacNeal, 1904.

Plasmodium praecox Grassi \& $\mathrm{Fe}$ letti, 1890.

Lucena, 1938b, S. Paulo, Brasil.

Trypanosoma sp.

Lucena, 1938, S. Paulo, Brasil.

(TURDUS RUFIVENTRIS) $=$ TURDUS RUFIVENTRIS RUFIVENTRIS Vieillot.

Trypanosoma sp.

Toxoplasma avium Marullaz, 1913.

Carini \& Maciel, 1916, S. Paulo, Brasil.

TURDUS RUFIVENTRIS RUFIVENTRIS Vieillot.

Plasmodium praecox Grassi \& Feletti, 1890.

Plasmodium vaughani Novy \& MacNeal, 1904.

Lucena, 1938b, S. Paulo, Brasil.

Trypanosoma sp.

Lucena, 1938b, S. Paulo, Brasil. 
TURDUS RUFIVENTRIS RUFIVENTRIS Vieillot.

Vide PLANESTICUS RUFIVENTRIS

TURDUS RUFIVENTRIS

(TYRANNUS MELANCHOLICUS) = TYRANNUS MELANCHOLICUS MELANCHOLICUS Vieillot.

Trypanosoma sp.

Carini \& Maciel, 1916, S. Paulo, Brasil.

Haemoproteus sp.

Mazza, Gonzalez, Franke, Gonzalez \& Alvarado, 1927, Argentina.

TYRANNUS MELANCHOLICUS MELANCHOLICUS Vieillot. Vide TYRANNUS MELANCHOLICUS.

TYTO ALBA TUIDARA (Gray). Vide STRIX FLAMMEA

(TYTO PERLATA $)=$ TYTO ALBA TUIDARA (Gray).

Haemoproteus danilewskyi Kruse 1890.

Iturbe \&: Gonzalez, 1916, Venezuela.

(URUBITINGA ALBICOLLIS) $=$ LEUCOPTERNIS ALBICOLLIS ALBICOLLIS (Latham).

Haemoproteus sp.

Brimont, 1909 e 1912, Guiana Franceza.

(VIREO CHIVI) $=$ VIREO CHIVI CHIVI (Vieillot).

Haemoproteus sp.

Carini \& Maciel, 1916, S. Paulo, Brasil.

VIREO CHIVI CHIVI (Vieillot). Vide VIREO CHIVI.

(VOLATINIA JACARINA) = VOLATINIA JACARINIA JACARINIA (Linnaeus).

Toxoplasma sp.

Lucena, 1938, S. Paulo, Brasil.

Toxoplasma avium Marullaz, 1913.

Carini \& Maciel, 1916, S. Paulo, Brasil.

VOLATINIA JACARINA JACARINA (Linnaeus), Vide VOLATINIA JACARINA.
(XIPHOCOLAPTES MAJOR) $=$ XIPHOCOLAPTES MAJOR CASTANEUS Ridgway.

Trypanosoma franchinianus Mazza \& Fiora, 1932.

Mazza \& Fiora, 1932, Argentina.

Haemoproteus sp.

Mazza \& Fiora, 1930a, Argentina.

XIPHOCOLAPTES MAJOR CASTA-

NEUS Ridgway. Vide XIPHOCO-

LAPTES MAJOR.

XOLMIS CINEREA Vieillot. Vide TAENIOPTERA NEUGETA.

$($ ZENAIDA AURICULATA $)=$ ZENAIDA AURICULATA VIRGATA Bertoni.

Haemoproteus sp.

Mazza, Deautier \& Steullet, 1930, Argentina.

Mazza, Gonzalez, Franke, Gonzalez \& Alvarado, 1927, Argentina.

ZENAIDA AURICULATA VIRGATA Bertoni. Vide ZENAIDA AURICULATA.

(ZONOTRICHIA PILEATA $)=$ BRACHYSPIZA CAPENSIS MATUTINA (Lichtenstein).

Trypanosoma sp.

Lutz \& Meyer, 1908, S. Paulo, Brasil.

Trypanosoma zonotrichiae Splendore, 1910.

Splendore, 1910, S. Paulo Brasil.

Haemoproteus sp.

Lutz \& Meyer, 1908, S. Paulo, Brasil.

Splendore, 1910, S. Paulo, Brasil.

Plasmodium sp.

Lutz \& Meyer, 1908, S. Paulo, Brasil.

Splendore, 1910, S. Paulo, Brasil.

Carini \& Maciel, 1916, S. Paulo, Brasil.

(ZONOTHRICHIA STRIGICEPS DABBENEI) = AIMOPHILA STRIGICEPS DABBENEI (Hellmayr)

Haemoproteus sp.

Mazza, Franke \& Alvarado, 1928, Argentina. 
II PARTE

LISTA DOS PARASITAS COM REFERENCIA ÃS ESPECIES HOSPEDADORAS E INDICAÇĀO BIBLIOGRÁFICA

Gênero HAEMOPROTEUS Kruse, 1890

Haemoproteus sp.

ACCIPITER sp.

Lutz \& Meyer, 1908, S. Paulo, Brasil.

BRACHYSPIZA CAPENSIS MATUTINA (Lichtenstein).

Lutz \& Meyer, 1908, S. Paulo, Brasil.

Splendore, 1910, S. Paulo, Brasil.

Lucena, 1938, S. Paulo, Brasil.

CAIRINA MOSCHATA (Linnaeus).

Leger, 1918, Guiana Franceza.

CALOSPIZA SELEDON (P. L. S. Müller).

Carini \& Maciel, 1916, S. Paulo, Brasil.

CARIAMA CRISTATA (Linnaeuus).

Lutz \& Meyer, 1908, S. Paulo, Brasil.

CARPODACUS MEXICANUS (Müller).

Beltrán, 1940, Mexico.

CATHARTES AURA RUFICOLLIS Spix.

Scott, 1926, Brasil.

CERCHNEIS SPARVERIUS EIDOS (Peters).

Mazza, Franke \& Alvarado, 1928, Argentina.

Leger, 1918, Guiana Franceza.

COLUMBA sp.

Aragão, 1907, 1907a, 1908, Rio de Janeiro.

Leger, 1918, Guiana Franceza.

COLUMBA MACULOSA MACULOSA Temminck.

Mazza \& outros, 1927, Argentina.

COLUMBA PICAZURO PICAZURO Temminck.

Mazza \& outros, 1932, Argentina.

COLUMBA RUFINA RUFINA Temminck \& Knip.

Leger, 1918, Guiana Franceza.
COLUMBA RUFINA SYLVESTRIS (Vieillot).

Lucena 1938, S. Paulo, Brasil.

COLUMBIGALLINA TALPACOTI TALPACOTI (Temminck).

Aragão, 1916, Rio de Janeiro, Brasil.

Lucena 1938, S. Paulo, Brasil.

COLUMBINA PICUI PICUI (Temminck).

Mazza \& Fiora, 1932, Argentina.

Lucena, 1938, S. Paulo, Brasil.

CORYPHOSPINGUS CUCULATUS (Müller).

Mazza \& Outros, 1927, Argentina.

Lucena, 1938, S. Paulo, Brasil.

CRAX NIGRA Linnaeus.

Brimont, 1912, Guiana Franceza.

CRYPTURELLUS OBSOLETUS OBSOLETUS (Temminck).

Lutz \& Meyer, 1908, S. Paulo, Brasil.

CYANOCORAX CHRYSOPS CHRYSOPS (Vieillot).

Lutz \& Meyer, 1908, S. Paulo, Brasil.

Mazza \& Fiora, 1930a, Argentina.

DACNIS CAYANA CAYANA (Linnaeus).

Pessôa, 1935, S. Paulo, Brasil.

EUXENURA GALEATA (Molina).

Carini \& Maciel, 1916, S. Paulo, Brasil.

ICTERUS JAMACAII (Gmelin).

Aragão, 1916, Rio de Janeiro, Brasil.

LEPIDOCOLAPTES AUGUSTIROS-

TRIS AUGUSTIROSTRIS (Vieillot).

Mazza \& Outros, 1927, Argentina.

LEUCOPTERNIS ALBICOLIS ALBICOLIS (Lathan).

Brimont, 1909 e 1912, Guiana Franceza.

MACHETORNES RIXOSA RIXOSA (Vieillot).

Mazza \& Fiora, 1932, Argentina. 
MOLOTHRUS BADIUS BADIUS (Vieillot).

Mazza \& Outros, 1927, Argentina. MOLOTHRUS BONARIENSIS BONARIENSIS (Gmelin).

Lucena, 1938, S. Paulo, Brasil.

MUSCIVORA TYRANNUS (Linnaeus).

Carini \& Maciel, 1916, S. Paulo, Brasil.

MYCTERIA AMERICANA Linnaeus.

Lutz \& Meyer, 1908, S. Paulo, Brasil.

MYIOBORUS BRUNNEICEPS d'Orbigny.

Mazza \& Fiora, 1932, Argentina.

NICTICORAX sp.

Lutz \& Meyer, 1908, S. Paulo, Brasil.

NYSTALUS MACULATUS STRIATIPECTUS.

Mazza \& outros, 1927, Argentina. ODONTOPHRUS CAPUEIRA CAPUEIRA (Spix).

Lutz \& Meyer, 1908, S. Paulo, Brasil.

ORTALIS CANICOLLIS PANTANALENSIS Cherrie \& Reichenberger.

Mazza, Dautier \& Steullet, 1930, Argentina.

Mazza \& Fiora, 1932, Argentina.

Romaña, 1932, Angentina, «in» Mazza \& Fiora, 1932, Argentina.

OTUS CHOLIBA CHOLIBA (Vieillot).

Lutz \& Meyer, 1908, S. Patlo, Brasil.

Carini, 1920, S. Paulo, Brasil.

Mazza, Franke \& Alvarado, 1928, Argentina.

OTUS CHOLIBA CRUCIGERUS (Spix).

Leger, 1908, Guiana Franceza.

PACHIRHAMPHUS POLYCHOPTERUS POLYCHOPTERUS (Vieillot).

Carini \& Maciel, 1916, S. Paulo, Brasil.

PASSER DOMESTICUS (Linnaeus).

Petrochi \& Zuccarini, 1925, Argentina.

Vogelsang, «in» Cordero, 1928, Argentina.
(PATO DO MATO).

Lutz \& Meyer, 1908, S. Paulo, Brasil.

PENELOPE SUPERCILIARIS JACUPEMBA Spix.

Carini \& Maciel, 1916, S. Paulo, Brasil.

PENELOPE OBSCURA BRONZINA Hellmayr.

Lutz \& Meyer, 1908, S. Paulo, Brasil.

PENELOPE PILEATA Wagler.

Scott, 1926, América do Sul.

PIAYA sp.

Neiva \& Penna, 1916, Brasil.

PIONUS MENSTRUUS (Linnaeus).

Scott, 1926, América do Sul.

PIRANGA FLAVA FLAVA (Vieillot). Mazza \& Fiora, 1930a, e 1932, Argentina.

PITANGUS SULPHURATUS BOLIVIANUS (Lafresnaye).

Mazza, Steullet \& Dautier, 1932, Argentina.

PLATYPSARIS RUFUS RUFUS (Vieillot).

Carini \& Maciel, 1916, S. Paulo, Brasil.

Pessôa, 1935, S. Paulo, Brasil.

POLYBORUS PLANCUS BRASILIENSIS (Gmelin).

Carini \& Maciel, 1916, S. Paulo, Brasil.

RHYNOPTYNX CLAMATOR CLAMATOR (Vieillot).

di Primio, 1925, Rio de Janeiro, Brasil.

SCARDAFELLA SQUAMMATA SQUAMMATA (Lesson).

Neiva \& Penna, 1916, Brasil.

SETOCHALCIS RUFA RUFA (Boddaert).

Mazza \& Fiora, 1932, Argentina.

SPOROPHILA sp.

Lutz \& Meyer, 1908, S. Paulo, Brasil.

TANAGRA ATRICAPILLA Gmelin.

Leger, 1918, Guiana Franceza.

TANAGRA CYANOPTERA Vieillot.

Pessôa, 1935, S. Paulo, Brasil. 
THRAUPIS sp.

Lucena, 1938, S. Paulo, Brasil.

THRAUPIS BONARIENSIS (Gmelin).

Mazza \& outros, 1927, Argentina.

THRAUPIS SAYACA SAYACA (Linnaeus).

Carini \& Maciel, 1916, S. Paulo, Brasil.

TINAMUS sp.

Lutz \& Meyer, 1908, S. Paulo, Brasil.

TYRANUS MELANCHOLICUS MELANCHOLICUS Vieillot.

Mazza \& outros, 1927, Argentina.

TYTO ALBA TUIDARA (Gray).

Lutz \& Meyer, 1908, S. Paulo, Brasil.
VIREO CHIVI CHIVI (Vieillot).

Carini \& Maciel, 1916, S. Paulo, Brasil.

XIPHOCOLAPTES MAJOR CASTANEUS Ridgway.

Mazza \& Fiora, 1930, Argentina.

XOLMIS CINEREA Vieillot.

Carini \& Maciel, 1916, S. Paulo, Brasil.

ZENAIDA AURICULATA VIRGATA Bertoni.

Mazza \& outros, 1927, Argentina.

Mazza, Dautier \& Steullet, 1930, Argentina.

ZONOTRICHIA STRYGICEPS DABBENEI Hellmayr.

Mazza, Franke \& Alvarado, 1928, Argentina.

Haemoproteus sp., ou Plasmodium praecox Grassi \& Feletti, 1890.

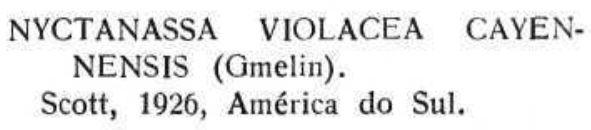

Haemoproteus columbae Celli \& Sanfelice, 1891.

COLUMBA sp.

Carini, 1912, S. Paulo, Brasil.

COLUMBA RUFINA SYLVESTRIS Vieillot.

Aragão, 1916, Rio de Janeiro, Brasil.

Lucena, 1939a, S. Paulo, Brasil.

di Primio, 1934, Rio Grande do Sul,

Brasil.

Haemoproteus danilewskyi Kruse, 1890.

AGELAIUS ICTEROCEPHALUS Lin- CAPSIEMPIS FLAVEOLA (Lichtenstein) naeus.

Plimmer, 1913, Mexico.

CALOSPIZA CYANOCEPHALA (P. L. S. Müller).

Plimmer, 1912, Brasil.

CALOSPIZA CYANOPTERA CYANOPTERA (Ser.).

Plimmer, 1912, América do Sul.

CALOSPIZA PERUVIANA (Desmarest).

Plimmer, 1912, Brasil.

CALOSPIZA SELEDON (P. L. S. Müller).

Plimmer, 1912, América do Sul. Iturbe \& Gonzalez, 1916, Venezuela.

CASSICULUS MELANICTERUS (Bonaparte).

Plimmer, 1912, Mexico.

CATHARTES AURA RUFICOLLIS Spix.

Darling, 1912, Panamá.

CHLOROPHONIA PYRRHOPHRYS (Sclater).

Iturbe \& Gonzalez, 1916, Venezuela. COLUMBIGALLINA TALPACOTI TALPACOTI (Temminck).

Iturbe \& Gonzalez, 1916, Venezuela. 
CROTOPHAGA ANI Linnaeus.

Iturbe \& Gonzalez, 1916, Venezuela.

CYANERPES CYANEA CYANEA (Linnaeus).

Plimmer, 1912 e 1913, América do Sul.

GERANOSPIZA CAERULESCENS CAERULESCENS (Vieillot).

Iturbe \& Gonzalez, 1916, Venezuela. MILVAGO CHIMACHIMA CHIMACHIMA (Vieillot).

Iturbe \& Gonzalez, 1916, Venezuela. MYIOZETETIS SIMILIS SIMILIS (Spix).

Iturbe \& Gonzalez, 1916, Venezuela.

PARABUTEO UNICINCTUS UNICINCTUS (Temminck).

- Iturbe \& Gonzalez, 1916, Venezuela.

PEZITES DEFILIPPII (Bonaparte).

Plimmer, 1913, Chile.

PITANGUS SULPHURATUS MAXIMILIANI (Cabanis \& Heine).

Plimmer, 1914, América do Sul. Iturbe \& Gonzalez, 1916, Venezuela.

RAMPHASTUS TOCO Müller. Iturbe \& Gonzalez, 1916, Venezuela.

RICHMONDEA PHOENICEA (Bona* parte).

Plimmer, 1917, Venezuela.

RUPORNIS LEUCORRHOUS (Gnoy \& Gaimard).

Iturbe \& Gonzalez, 1916, Venezuela.
SCOTOPELIA BOUVIERI (Sharpe). Plimmer, 1912, América do Sul.

SICALIS FLAVEOLA FLAVA (Müller).

Iturbe \& Gonzalez, 1916, Venezuela.

SYCALIS COLUMBIANA Cabanis.

Iturbe \& Gonzalez, 1916, Venezuela.

TANAGRA VIOLACEA VIOLACEA Linnaeus.

Iturbe \& Gonzalez, 1916, Venezuela.

THRAUPIS EPISCOPUS Linnaeus.

Plimmer, 1912, América do Sul.

THRAUPIS EPISCOPUS CANA (Ser.)

Iturbe \& Gonzalez, 1916, Venezuela.

THRAUPIS PALMARUM MELANOPTERA Sclater.

Iturbe \& Gonzalez, 1916, Venezuela.

THRAUPIS PALMARUM PALMARUM (Neuwied).

Plimmer, 1912, América do Sul.

TROGONURUS RUFUS RUFUS (Gmelin).

Iturbe \& Gonzalez, 1916, Venezuela.

TURDUS FUMIGATUS FUMIGATUS Lichtenstein.

Iturbe \& Gonzalez, 1916, Venezuela.

TURDUS LEUCOMELAS ALBIVENTER Spix.

Iturbe \& Gonzalez, 1916, Venezuela.

TYTO ALBA TUIDARA (Gray).

Iturbe \& Gonzalez, 1916, Venezuela.

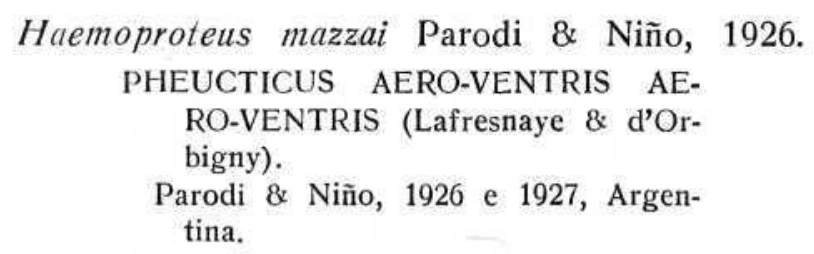

Haemoproteus mazzai Parodi \& Niño, 1926.

PHEUCTICUS AERO-VENTRIS AERO-VENTRIS (Lafresnaye \& d'Orbigny).

Parodi \& Niño, 1926 e 1927, Argentina.

Gênero LEUCOCYTOZOON Danilewskyi, 1890.

Leucocytozoon sp.

BOMBYCILLA CEDRORUM Vieillot, PIRANGA FLAVA FLAVA (Vieillot).

Beltrán, 1940, Mexico.

NOTIOPSAR CURAEUS (Molina).

Plimmer, 1915, Chile.

Mazza \& Fiora, 1930a, Argentina.

PITANGUS SULPHURATUS BOLIVIANUS (Lafresnaye).

OTUS CHOLIBA CHOLIBA (Vieillot).

Lutz \& Meyer, 1908, S. Paulo, Brasil.

Mazza \& Fiora, 1930a, Argentina.

SICALIS LUTEOLA LUTEIVENTRIS (Meyer).

Scott, 1926, Chile. 
Leucocytozoon lutzi Carini, 1920.

OTUS CHOLIBA CHOLIBA (Vieillot).

Carini, 1920, S. Paulo, Brasil.

Gênero PLASMODIUM Marchiafava \& Celli, 1885.

Plasmodium sp.

BRACHYSPIZA CAPENSIS MATUTI- MOLOTHRUS sp.

NA (Lichtenstein).

Beltrán, 1940, Mexico.

Lutz \& Meyer, 1908, S. Paulo, Brasil.

Splendore, 1910, S. Paulo, Brasil.

Carini \& Maciel, 1916, S. Paulo, Brasil.

CARPODACUS MEXICANUS (Müller).

Beltrán, 1940, Mexico.

COLAPTES CAMPESTRIS CAMPES-

TRIS (Vieillot).

Carini \& Maciel, 1916, S. Paulo, Brasil.

RHEA AMERICANA INTERMEDIA

Rothschild \& Chubb.

Carine \& Maciel, 1916, S. Paulo, Brasil.

(SARACURA).

Lutz \& Meyer, 1908, S. Paulo, Brasil.

SYNALLAXIS RUFICAPILLA Vieillot.

Carini \& Maciel, 1916, S. Paulo, Brasil.

Plasmodium cathemerium Hartman, 1927.

BRACHYSPIZA CAPENSIS MATUTI- MOLOTHRUS BONARIENSIS BONANA (Lichtenstein).

Lucena, 1938a, S. Paulo, Brasil. RIENSIS (Gmelin).

MIMUS SATURNINUS MODULATOR (Gould). Lucena, 1938c, S. Paulo Brasil. PASSER DOMESTICUS (Linnaeus). Lucena, 1938a, S. Paulo, Brasil.

Lucena, 1939a, S. Paulo, Brasil.

Plasmodium elongatum Huff, 1930.

BRACHYSPIZA CAPENSIS MATUTINA (Lichtenstein).

Lucena, 1938a, S. Paulo, Brasil.

Plasmodium lutzi Lucena, 1938.

ARAMIDES CAJANEA CAJANEA

(Müller).

Lucena, 1938d, 1939 e 1939a, S. Paulo, Brasil.

Plasmodium nucleophilum Manwell, 1935.

BRACHYSPIZA CAPENSIS MATUTI- PASSER DOMESTICUS (Linnaeus),

NA (Lichtenstein).

Lucena, 1939a, S. Paulo, Brasil.

Lucena, 1938b, S. Paulo, Brasil. 
Plasmodium praecox Grassi \& Feletti, 1890.

APHELOCOMA SORDIDA (Swainson). Plimmer, 1914, Mexico.

ARA MACAO (Linnaeus).

Plimmer, 1912, América Central.

CALOSPIZA DESMARESTI (Vieillot). Plimmer, 1912, Brasil.

CALOSPIZA FASTUOSA (Lesson). Scott, 1926, Brasil.

CARPODACUS MEXICANUS (Müller). Plimmer, 1912, Mexico.

DACNIS CAYANA CAYANA (Linnaeus).

Plimmer, 1912, América do Sul.

EUETHIA CANORA (Gmelin).

Plimmer, 1912, Cuba.

ICTERUS JAMACAII (Gmelin).

Plimmer, 1912, Brasil.

PASSER DOMESTICUS (Linnaeus).

Petrochi \& Zuccarini, 1925, Argentina.

PASSERINA LECLANCHERI Lafresnaye \& Vieillot.

Plimmer, 1912, Mexico.
PYGOCHELIDON CYANOLEUCUS CYANOLEUCUS (Vieillot).

Iturbe \& Gonzalez, 1916, Venezuela.

RHAMPHOCELUS BRESILIUS BRESILIUS (Linnaeus).

Scott, 1926, Brasil.

SCARDAFELLA SQUAMMATA SQUAMMATA (Leson).

Plimmer, 1912, América do Sul.

SERINUS CANARIUS Linnaeus.

Reis \& Nobrega, 1936, S. Paulo, Brasil.

TANAGRA VIOLACEA VIOLACEA Linnaeus.

Iturbe \& Gonzalez, 1916, Venezuela.

TURDUS LEUCOMELAS LEUCOMELAS Vieillot.

Lucena, 1938b, S. Paulo, Brasil.

TURDUS RUFIVENTRIS RUFIVENTRIS Vieillot.

Lucena, 1938b, S. Paulo, Brasil.

\title{
Plasmodium vaughani Novy \& MacNeal, 1904.
}

\author{
ARAMIDES CAJANEA CAJANEA TURDUS LEUCOMELAS LEUCOME- \\ (Müller). \\ LAS Vieillot. \\ Lucena, 1939a, S. Paulo, Brasil. \\ THAMNOPHILUS RUFICAPILLUS \\ RUFICAPILLUS Vieillot. \\ Lucena, 1939a, S. Paulo, Brasil. \\ Lucena, 1938b, S. Paulo, Brasil. \\ TURDUS RUFIVENTRIS RUFIVEN- \\ TRIS Vieillot. \\ Lucena, 1938b, S. Paulo, Brasil. \\ TURDUS CHIGUANCO ANTHRACI- \\ NUS (Burmeister). \\ Mazza \& Fiora, 1930, Argentina.
}

Gênero TOXOPLASMA Nicolle \& Manceaux, 1909. Toxoplasma sp.
ARAMIDES CAJANEA CAJANEA COLUMBIGALLINA TALPACOTI TAL- (Müller).
PACOTI (Temminck).
Lucena, 1938, S. Paulo, Brasil.
Lucena, 1938, S. Paulo, Brasil.
BRACHYSPIZA CAPENSIS MATUTI- NA (Lichtenstein).
CORYPHOSPINGUS CUCULATUS
(Müller).
Lucena, 1938, S. Paulo, Brasil.
Lucena, 1938, S. Paulo, Brasil.
COLUMBA RUFINA SYLVESTRIS Vieillot.
GALLUS GALLUS DOMESTICUS Lin- naeus.
Lucena, 1938, S. Paulo, Brasil.
Lucena, 1938, S. Paulo, Brasil.


Rev. Fac. Med. Vet. S. Paulo - Vol. 2, fasc. 1, 1941

MOLOTHRUS BONARIENSIS BONA- THRAUPIS SAYACA SAYACA (LinRIENSIS (Gmelin). naeus).

Lucena, 1938, S. Paulo, Brasil.

Pessôa, 1929, S. Paulo, Brasil.

SPOROPHILA CAERULESCENS Vi- VOLATINIA JACARINA JACARINA eillot.

Lucena, 1938, S. Paulo, Brasil. (Linnaeus).

Lucena, 1938, S. Paulo, Brasil.

TACHYPHONUS RUFUS CORONA-

TUS Vieillot.

Pessôa, 1929, S. Paulo, Brasil.

Toxoplasma avium Marullaz, 1913.

BRACHYSPIZA CAPENSIS MATUTI- SARCORAMPHUS PAPA (Linnaeus). NA (Lichtenstein).

Carini \& Maciel, 1916, S. Paulo, Brasil.

ELAENIA ALBICEPS ALBICEPS (Lafresnaye \& d'Orbigny).

Carini \& Maciel, 1916, S. Paulo, Brasil.

Carini \& Maciel, 1916, S. Paulo, Brasil.

? SERINUS CANARIUS Linnaeus.

Rosenbusch, 1912, Argentina.

SPOROPHILA ALBOGULARIS (Spix).

Carini \& Maciel, 1916, S. Paulo, Brasil.

GNORIMOPAR CHOPI CHOPI (Vieillot).

Carini \& Maciel, 1916, S. Paulo, Brasil.

PITANGUS SULPHURATUS MAXIMILIANI (Cabanis \& Heine).

Carini \& Maciel, 1916, S. Paulo, Brasil.

PYGOCHELIDON CYANOLEUCUS CYANOLEUCUS (Vieillot).

THRAUPIS PALMARUM PALMARUM (Neuwied).

Carini \& Maciel, 1916, S. Paulo, Brasil.

TURDUS RUFIVENTRIS RUFIVENTRIS Vieillot.

Carini \& Maciel, 1916, S. Paulo, Brasil.

VOLATINA JACARINA JACARINA (Linnaeus).

Carini \& Maciel, 1916, S. Paulo, Carini \& Maciel, 1916, S. Paulo, Brasil. Brasil.

Toxoplasma gondii (Nicolle \& Manceaux, 1908).

COLUMBA sp.

Carini, 1911, S. Paulo, Brasil.

Arantes, 1914, Rio de Janeiro, Brasil.

Carini \& Maciel, 1916, S. Paulo, Brasil.

Toxoplasma sicalidis (Aragão, 1911) Nöller, 1920.

SICALIS FLAVEOLA FLAVA (Müller).

Aragão, 1911, Rio de Janeiro, Brasil.

Toxoplasma sporophilae Nöller, 1920.

SPOROPHILA ALBOGULARIS (Spix).

Nöller, 1920, «in» Wenyon, 1926. 
Gênero HAEMOGREGARINA Danilewskyi, 1885.

Haemogregarina sp.

BELONOPTERUS CHILENSIS LAM- SALTATOR SIMILIS Lafresnaye \& PRONOTUS (Wagler).

Neiva \& Penna, 1916, Brasil. d'Orbigny.

Lucena, 1938, S. Paulo, Brasil.

CERTHIAXIS CINNAMOMEA RUS- TACHYPHONUS RUFUS CORONASEOLA (Vieillot). TUS (Vieillot).

Lucena, 1938, S. Paulo, Brasil.

Lucena, 1938, S. Paulo, Brasil.

CYANOCOMPSA CYANEA CYANEA (Linnaeus).

Lucena, 1938, S. Paulo, Brasil. THRAUPIS SAYACA SAYACA (Linnaeus).

Pessôa, 1929, S. Paulo Brasil.

MOLOTHRUS BONARIENSIS BONA.

RIENSIS (Gmelin).

Corrêa, 1938, S. Paulo, Brasil.

Haemogregarina aragãoi di Primio, 1925.

PAROARIA CAPITATA CAPITATA

(Lafresnay \& d'Orbigny).

di Primio, 1925, Rio de Janeiro, Brasil.

Haemogregarina atticorae Aragão, 1911.

PYGOCHELIDON CYANOLEUCUS CYANOLEUCUS (Vieillot).

Aragão, 1911, Rio de Janeiro, Brasil.

Haemogregarina brachyspizae, Aragão, 1911.

BRACHYSPIZA CAPENSIS MATUTI-

NA (Lichtenstein).

Aragão, 1911, Rio de Janeiro, Brasil.

Haemogregarina paroariae Aragão, 1911.

PAROARIA DOMINICANA (Linnaeus).

Aragão, 1911, Rio de Janeiro, Brasil.

Haemogregarina paulasousai Corrêa, 1928.

STEPHANOPHORUS DIADEMATUS

(Temminck).

Corrêa, 1928, S. Paulo, Brasil.

Haemogregarina pessoai Corrêa, 1928.

POOSPIZA THORACICA (Nordmann).

Corrêa, 1928, S. Paulo, Brasil. 
Haemogregarina pintoi di Primio, 1925.
CATHARTES AURA RUFICOLLIS CORAGYPS ATRATUS FOENTENS Spix.
di Primio, 1925, Rio de Janeiro, Bra- di Primio, 1925, Rio de Janeiro, sil.

Haemogregarina rhamphocoeli Aragão, 1911.

RHAMPHOCELUS BRESILIUS BRE-

SILIUS (Linnaeus).

Aragão, 1911, Rio de Janeiro, Brasil.

Haemogregarina sporophilae Aragão, 1911.

SPOROPHILA ALBOGULARIS (Spix).

Aragão, 1911, Rio de Janeiro, Bra-

sil.

Haemogregarina tanagrae Aragão, 1911.

THRAUPIS PALMARUM PALMARUM

(Neuwied).

Aragão, 1911, Rio de Janeiro, Brasil.

Haemogregarina travassosi di Primio, 1925.

TARABA MAJOR MAJOR (Vieillot).

di Primio, 1925, Rio de Janeiro,

Brasil.

Gênero TRYPANOSOMA Gruby, 1843.

Trypanosoma sp.

ARCHIPLANUS ALBIROSTRIS (Vi- CHAMAEZA BREVICAUDA BREVIeillot).

Carini \& Maciel, 1916, S. Paulo, Brasil. CAUDA.

Carini \& Maciel, 1916, S. Paulo, Brasil.

BATARA CYNEREA CYNEREA (Vi- COCCYZUS MELACORYPHUS, Vieileillot).

Carini \& Botelho, 1914, S. Paulo, Brasil. lot.

Mazza, Franke \& Gonzalez, 1927, Argentina.

BRACHYSPIZA CAPENSIS MATUTINA (Lichtenstein).

GNORIMOPAR CHOPI CHOPI (Vieillot).

Lutz \& Meyer, 1908, S. Paulo, Bra- Carini \& Maciel, 1916, S. Paulo, sil. Brasil.

BUTORIDES STRIATUS STRIATUS (Linnaeus).

Leger, 1918, Guiana Franceza.

Migone, 1916, Paraguai.

GRALLARIA VARIA IMPERATOR Lafresnaye.

Carini \& Botelho, 1914, S. Paulo, Brasil. 
HARPIA HARPYJA (Linnaeus).

Iturbe \& Gonzalez, 1916, Venezuela. MOLOTHRUS BADIUS BADIUS (Vieillot).

Mazza, Franke \& Gonzalez, 1927, Argentina.

MYCTERIA AMERICANA Linnaeus.

Migone, 1916, Paraguai.

OTUS CHOLIBA CHOLIBA (Vieillot)

Lutz \& Meyer, 1908, S. Paulo, Brasil.

PIAYA CAYANA MACROURA Gambel.

Carini \& Botelho, 1914, S. Paulo, Brasil.

PIRANGA FLAVA FLAVA (Vieillot). Mazza \& Fiora, 1930e, Argentina.

PITANGUS SULPHURATUS MAXIMILIANI (Cabanis \& Heine).

Carini \& Botelho, 1914, S. Paulo, Brasil,
THERISTICUS CAUDATUS CAUDATUS (Boddaert).

Migone, 1916, Paraguai.

THRAUPIS ORNATA (Sparrmann).

Cerqueira, 1906, Rio de Janeiro, Brasil.

TITYRA CAYANA BRASILIENSIS (Swainson).

Pessôa, 1935, S. Paulo, Brasil.

TURDUS LEUCOMELLAS LEUCOMELLAS Vieillot.

Lucena, 1938, S. Paulo, Brasil.

TURDUS RUFIVENTRIS RUFIVENTRIS Vieillot.

Carini \& Maciel, 1916, S. Paulo, Brasil.

Lucena, 1938, S. Paulo, Brasil.

Pessôa, 1935, S. Paulo, Brasil.

TYRANNUS MELANCHOLICUS MELANCHOLICUS Vieillot.

Carini \& Maciel, 1916, S. Paulo, Brasil.

Trypanosoma ardea Leger, 1918.

NYCTANASSA VIOLACEA CAYEN-

NENSIS (Gmelin).

Leger, 1918, Guiana Franceza.

Trypanosoma ardea major Leger, 1918.

FLORIDA CAERULEA (Linnaeus). Leger, 1918, Guiana Franceza.

Trypanosoma avium Danilewskyi, 1885.

ICTERUS JAMACAII (Gmelin).

Plimmer, 1914, Brasil.
LEISTES MILITARIS MILITARIS (Linnaeus).

Plimmer, 1912, Guiana Ingleza.

Trypanosoma avium minus Danilewsky, 1888.

FLORIDA CAERULEA (Linnaeus).

Cerqueira, 1906, Rio de Janeiro, Brasil.

LEUCOPHOYX THULA THULA (Molina).

Cerqueira, 1906, Rio de Janeiro, Brasil.
NYCTICORAX NYCTICORAX HOACTLI (Gmelin).

Cerqueira, 1906, Rio de Janeiro, Brasil.

Aragão, «in» Cerqueira, 1906, Brasil. 
Trypanosoma balfouri Mazza, Oliva, Schürmann \& Gutdeutsch, 1932.

PAROARIA CORONATA (Müller). Mazza, Oliva, Schürmann \& Gutdeutsch, 1932, Argentina.
PSITTACARA ACUTICAUDATA ACUTICAUDATA (Vieillot).

Mazza, Oliva, Schürmann \& Gutdeutsch, 1932, Argentina.

Trypanosoma catharistae Mesnil, 1912.

CORAGYPS ATRATUS FOETENS

(Lichtenstein).

Brimont, 1909 e 1912, Guiana Franceza.

Mesnil, 1912, Guiana Franceza.

Trypanosoma dabbenei Mazza, Deautier \& Steullet, 1927.

CHAMAEZA BREVICAUDA BREVI-

CAUDA (Vieillot).

Mazza, Deautier \& Steullet, 1927, Argentina.

Trypanosoma franchinianus Mazza \& Fiora, 1932.

XIPHOCOLAPTES MAJOR CASTA-

NEUS Ridgway.

Mazza \& Fiora, 1930a, e 1932, Argentina.

Trypanosoma mesnili guyanense Mesnil, 1912.

HETEROSPIZIAS MERIDIONALIS

MERIDIONALIS (Latham).

Brimont, 1912, Guiana Franceza.

Mesnil, 1912, Guiana Franceza.

Trypanosoma langeronianus Mazza \& Fiora, 1932.

CERCHNEIS SPARVERIUS EIDOS

(Peters),

Mazza \& Fiora, 1930a e 1932, Argentina.

Trypanosoma pedrozi Carini \& Botelho, 1914.

CRAX FASCIOLATA Spix.

Carini \& Botelho, 1914, S. Paulo, Brasil.

Trypanosoma schistochlamydis Splendore, 1910.

SCHISTOCHLAMYS RUFICAPILLUS

CAPISTRATUS (Neuwied).

Splendore, 1910, S. Paulo, Brasil. 
D. T. Lucena - Protozoários hemoparasitas de aves

Trypanosoma tinami Mesnil, 1912.

CRYPTURELLUS CINEREUS (Gme- TINAMUS MAJOR MAJOR (Gmelin).

lin).

Brimont, 1912, Guiana Franceza.

Brimont, 1912, Guiana Franceza.

Mesnil, 1912, Guiana Franceza.

Mesnil, 1912, Guiana Franceza.

Trypanosoma zonotrichia Splendore, 1910.

BRACHYSPIZA CAPENSIS MATUTI-

NA (Lichtenstein).

Splendore, 1910, S. Paulo, Brasil.

\section{PARTE \\ LISTA DAS ESPECIES DOS PROTOZOÃRIOS HEMOPARASITAS MENCIONADOS}

Gênero HAEMOPROTEUS Kruse, 1890.

Haemoproteus danilewskyi Kruse, 1890. Haemoproteus mazzai Parodi \& Niño, Haemoproteus columbae Celli \& Sanfe1926.

lice, 1891.

\section{Gênero LEUCOCYTOZOON Danilewskyi, 1890.}

Leucocytozoon lutzi Carini, 1920.

Gênero PLASMODIUM Marchiafava \& Celli, 1885.

Plasmodium praecox Grassi \& Feletti, Plasmodium elongatum Huff, 1930. 1890.

Plasmodium vaughani Novy \& MacNeal, 1904.

Plasmodium nucleophilum Manwell, 1930.

Plasmodium lutzi Lucena, 1938.

Plasmodium cathemerium Hartman, 1927.

Gênero TOXOPLASMA Nicolle \& Manceaux, 1909.

Toxoplasma gondii (Nicolle \& Man- Toxoplasma avium Marullaz, 1913.

ceaux, 1908). Toxoplasma sporophilae Nöller, 1920.

Toxoplasma sicalidis (Aragão, 1911)

Nöller, 1920.

\section{Gênero HAEMOGREGARINA Danilewskyi, 1885.}

Haemogregarina atticorae Aragão, 1911.

Haemogregarina brachyspizae Aragão, 1911.

Haemogregarina paroariae Aragão, 1911.

Haemogregarina rhamphocoeli Aragão, 1911.
Haemogregarina sporophilae Aragão, 1911.

Haemogregarina tanagrae Aragão, 1911.

Haemogregarina aragãoi di Primio 1925.

Haemogregarina pintoi di Primio, 1925. 
Haemogregarina travassosi di Primio, Haemogregarina paulasousai Corrêa, 1925. 1928.

Haemogregarina pessôai Corrêa, 1928.

\section{Gênero TRYPANOSOMA Gruby, 1843.}

Trypanosoma avium Danilewsky, 1885. Trypanosoma ardea Leger, 1918.

Trypanosoma avium minus Danilewsky, Trypanosoma ardea major Leger, 1918. 1888.

Trypanosoma schistochlamydis Splendore, 1910.

Trypanosoma catharistae Mesnil, 1912.

Trypanosoma mesnili guyanense Mesnil, 1912.

Trypanosoma tinami Mesnil, 1912.

Trypanosoma pedrozi Carini \& Botelho 1914.

Trypanosoma dabbenei Mazza, Dautier

\& Steullet, 1927.

Trypanosoma balfouri Mazza, Oliva, Schürmann \& Gutdeutsch, 1932.

Trypanosoma franchinianus Mazza \& Fiora, 1932.

Trypanosoma langeronianus Mazza \& Fiora, 1932.

\section{BIBLIOGRAFIA}

ARAgão, H. B. - 1907 - «Sobre o ciclo evolutivo do halterídio do pombo». Rio. - - - 1907a - «Sobre o ciclo evolutivo do halterídio do pombo". Rio.

- $\quad$ - 1908 - «Über den Entwicklungsgang und die Obertragung von Haemoproteus columbae». Arch. Protist. 12; 154.

- - 1911 "Observações sobre algumas hemogregarinas das aves». Mem. Ist. Osw. Cruz, 3 (1) 54.

- $\quad 1916$ - «Pesquiza sobre o Haemoproteus columbae». BrasilMédico, 30 (45) (46), 353 e 361.

- $\quad 1918$ - «Classificação dos Hemosporidios». Mem. Inst. Butantan, 1: 167-184.

- 1935 - «Sensibilité des qualques oiseaux brésiliens au Plasmodium praecox». Compt. Rend. Soc. Biol., 118 (9) 903-904.

Arantes, J. B. - 1914 - "Toxoplasmose. Evolução do Toxoplasma canis no sistema nervoso do pombo e as lesões por ele produzidas». Brasil-Médico, 28 (15) 144.

- 1914a - «I. Infecções experimentais pelo Toxoplasma. II. Novas localizações deste protozoário. III. A bipartição é o seu único processo de multiplicação (2.a nota preliminar)". BrasilMédico, 28 (40) 373.

- $\quad$ - 1914b - "Contribuição para o estudo do Toxoplasma. These. Rio. Tip. Jornal do Comercio.

BACMANN, A. - 1916 - «Nota previa sobre el hematozoario de los fringílidos». Prensa Med. Argentina, 3 (16) 165.

Beltrán, E. - 1940 - «Examen protozoológico de la sangre de algunos pajaros mexicanos». Ciencia, 1 (1) 20-21.

Brimont, E. - 1909 - «Sur qualques hématozoaires de la Guyane. 1ère. note». Compt. Rend. Soc. Biol., 67: 169-171.

1912 "Tripanosomes d'oiseaux de la Guyane». Compt. Rend. Soc. Biol., 72: 884-888. 
CARINI, A. - 1909 - «Infection spontanée du pigeon et du chien due au Toxoplasma cuniculi». Bull. Soc. Pathol. Exot., 2: 465.

- 1911 - «Infection spontanée du pigeon et du chien due au Toxoplasma caniculi». Bull. Soc. Path. Exot, 4 (8) 518-519.

- 1912 "Sur un nouvel hématozoaire du pigeon». Compt. Rend. Soc. Biol. 73: 396.

- 1920 "Sur un Leucocytozoon d'une chouette du Brésil». Bull. Soc. Path. Exot., 13 (7) 506-508.

Carini, A. \& Botelho, C. - 1914 - «Alguns Trypanosomas de Passaros do Brasily. An. Paul. Med. Cirur., 3 (2) 1-3.

CARINI, A. \& MACIEL, J. - 1916 - "Quelques hémoparasites d'oiseaux du Brésil». Bull. Soc. Path. Exot., 9 (4) 247-265.

Cerqueira, A. D. C. - 1906 - "Contribuição ao estudo dos Trypanosomas das aves». These. Rio. L. Malafaia Jr,

Contney, G. R. - 1936 - "A Check-List and Host-Index of the Genus Haemoproteus. Jour. Parasit., 22 (1) 68-105.

- $\quad 1937$ « Catalog and Host-Index of the Genus Leucocytozoon». Jour. Parasit., 23 (2) 202-212.

Coatney, G. R. \& Roudabush. R. L. - 1936 - "A Catolog and Host-Index of the Genus Plasmodium». Jour. Parasit., 22 (4) 338-353.

Cordero, E. H. - 1928 - «Protozoarios parásitos de algunos animales del Uruguay". Bol. Inst. Clin. Quirurgica, 1: 586.

CoRrea, C. 1928 - «Contribuição ao estudo das hemogregarinas do Brasil». Rev. Biol. Hyg., 1 (3) 75-81.

Darling, S. T. - 1912 - «Some blood parasites (Haemoproteus and Haemogregarina». Bull. Soc. Path. Exot., 5 (2) 71-73.

FonsecA, J. A. B. - 1938 - «Infeção experimental do Culex quinquefasciatus Say, $1823 \mathrm{com}$ Plasmodium praecox (P. relictum) Grassi \& Feletti, 1890\%. Rev. Biol. Hig., 9 (1) 1-5.

Godoy, A. \& LACORTE, J. G. - 1928 - «Action d'un noyau de l'oxy-aminoquinoléine sur les gamètes et les sporozoites de l'halteridium du pigeon». Compt. Rend. Soc. Biol., 98: 617-619.

Hamerton, A. E. - 1929 - «Report on the deaths occurring in the Society's Gardens during the year 1928». Proc. Zool. Soc., 49-59.

- 1930 - «Report on the deaths occurring in the Society's Gardens during the year 1929. Proc. Zool. Soc, 357-380.

IHERINa, R. VON - 1930 - «A entomophagia do Tico-Tico (Brachyspiza capensis)». Rev. Biol. Hig., 2 (2) 151.

IHERina, H. von \& IHERino, R. von - 1907 - "Catalogos da Fauna Brasileira». S. Paulo. Tip. Diario Oficial.

Iturbe, J. \& Gonzalez, E. - 1916 - «El paludismo de las aves en Venezuela». Caracas.

- 1921 "Contribuicion del Laboratorio Iturbe en el 3er. Congresso Venezuelano de Medicina: Sobre algunos datos de Protozoologia y Parasitologia recogidos en San Juan de los Morros". Caracas.

Leoer, M. - 1918 - «Parasites sanguicoles d'oiseaux de la Guyane». Bull. Soc, Path. Exot., 11 (2) 124-130.

Lucena, D. T. - 1938 - «Haemoparasitas de algumas aves de São Paulo», Rev. Biol. Hig., 9 (2) 158-161. 
LucenA, D. T. - 1938a - «Malaria aviaria. II. - Plasmodium cathemerium Hartman, 1927, parasita do tico-tico ( $B$. pileata) e do pardal ( $P$. domesticus) e $P$. elongatum Huff, 1930, parasita do ticotico, em São Paulo». A Folha Médica, 19 (20) 233-238.

- $\quad$ - 1938b - «Malaria aviaria. III - Da presença dos Plasmodium praecox, nucleophilum e vaughani em passaros de São Paulo». A Folha Médica, 19 (34) 404-406.

- $\quad$ - 1938c - «Malaria aviaria. IV - Infecção experimental do Culex fatigans Wiedmann, 1828 e do Anopheles strodei Root, 1926, pelo Plasmodium cathemerium Hartman, 1927». Rev. Biol. Hig., 9 (1) $47-50$.

- - 1938d - «Malaria aviaria. I - Plasmodium lutzi n. sp., parasita da Saracura (Aramides c. cajanea Müller)». Rev. Ass. Paul. Med. 13 (1) 46 e Ann. Paul. Med. Cirur., 36 (5) 507.

- 1939 - «Malaria aviaria. I - Plasmodium lutzi n. sp.," parasita da Saracura (Aramides c. cajanea Müller)». Bol. Biol., (nova serie) 4 (1) 27-31.

- 1939a - «Malaria aviaria. Subsidios para sua sistemática e transmissão». Recife Tip. Jornal do Comercio.

Lutz, A. \& Meyer, C. $-1908-$-Hematozoarios endoglobulares». Rev. Med. S. Paulo (9) 3-16.

MazzA, S. \& Fiora, A. - 1930 - «Proteosoma de mirlo, Planesticus anthracinus (Burm.) y Leucocytozoon de benteveo, Pitangus sulphuratus bolivianus (Lafr.) y fugero, Piranga flava (Vieill.) de Tumbaya, Jujuy.» 5.a Reun. Soc. Arg. Patol. Reg. Norte, 2: 993-995.

- $\quad$ - 1930a - «Hemoparasitos de un grupo de aves de Tumbaya y Zapla. (Jujuy)». 5.a Reun. Soc. Arg. Patol. Reg. Norte, 2.

- 1932 - «Nuevos hemoproteus y microfilarias de aves de Jujuy». 7.a Reun. Soc. Arg. Patol. Reg. Norte, 998-1001.

Mazza, S. Deautier, E. \& Steullet, A. - 1927 - "Investigación de hemoparásitos en algunas aves de Missiones". Bol. Inst. Clin. Quirurg. B. Aires, 365-368.

- $\quad-1930$ - «Parasitos de la sangre de algunas aves de «Los Talas» (Buenos-Aires) y de otras en cautividad em Buenos-Aires". 5.a Reun. Soc. Arg. Patol. Reg. Norte, 2: 996-998.

Mazza, S., Franke, I. \& Alvarado, S. - 1928 - "Nuevos hemoproteus de aves del norte». Bol. Inst. Clin. Quirurg. B. Aires, 605-606.

Mazza, S., Franke, I. \& Gonzalez, J. M. L. - 1927 - «Tripanosoma de pájaros del norte». Bol. Inst. Clin. Quirurg. B. Aires, 369-371.

Mazza, S, Steullet, A. \& Deautier, E. - 1932 - «Hemoparásitos de un grupo de aves de Escobar y Sierra de la Ventana (Prov. de BuenosAires) y de la ciudad de Buenos-Aires». 7.a Reun. Soc. Arg. Patol. Reg. Norte, 1002-1004.

Mazza, S., Gonzalez, C., Franke, I., Gonzalez, J. M. \& Alvarado, S. - 1927 - «Haemoproteus de pájaros del norte». Bol. Inst. Clin. Quirurg. B. Aires, 360-364.

Mazza, S., Oliva, C., Schürmann, K. \& Gutheutsch, H. - 1932 - «Parásitos de la sangre de algunas aves de la zona del Chaco». 7.a Reun. Soc. Arg. Patol. Reg. Norte, 1005-1007. 
MesniL, T. - 1912 - Notas ao trabalho de Brimont (1912): "Tripanosomes d'oiseaux de la Guyane». Compt. Rend. Soc. Biol., 72: 884-888.

Migone, L. E. - 1916 - «Parasitologie de certains animaux du Paraguay». Bull. Soc. Path. Exot., 9: 359.

MIRANDA RibeIRo, A. - 1938 - «Notas ornithologicas. XIII: - Tinamidae». Rev. Museu Paul., 23: 667-681.

Neiva, A. \& Penna B. - 1916 - «Viagem cientifica pelo Norte da Bahia, sudoeste de Pernambuco, sul do Piauí e de Norte a sul de Goiaz», Mem. Inst. Osw. Cruz, 8 (3) 74-224.

PARodi, S. E. \& Niño, F. L. - 1926 - «Haemoproteus de la sangre periférica del Pheucticus aureiventris». Prensa Med. Argent., 13 (15) 502-503.

- 1927 - «Haemoproteus n. sp. de la Reina Mora del Norte (Pheucticus aureiventris d'Orb. \& Lafr. fringílido)". Bol. Inst. Clin. Quirurg. B. Aires, 356-359.

PessôA, S. B. - 1935 - «Novos hemoparasitas de aves do Brasil». Ann. Fac. Med. Univ. S. Paulo, 11 (2) 3-5.

PessôA, S. B. \& Correa, C. $-1929-$ «Nota sobre Toxoplasma dos passaros». Ann. Paul. Med. Cirur., 17 (20).

Petrochi, J. \& Zuccarini, J. A. - 1925 - «Sobre la presencia del Plasmodium danilewski y de Haemoproteus sp. en la sangre de los gorriones (Passer domesticus) de Buenos Aires». Rev. Inst. Bact. Dep. Hig., 4 (1) 57-63.

Pinto, Cesar - 1935 - «Protozoarios observados no Brasil». Mem. Inst. Osw. Cruz, 18 (1) 233-238.

- 1933 - «Profilaxia das doenças infecciosas e parasitarias dos animais domésticos do Brasil». Rio. Tip. Minist. Agricult.

- 1938 - «Zoo-Parasitos de interesse médico e veterinario». Rio. Tip. Pimenta de Mello.

Pinto, Oliverio M. O. - 1935 - «Aves da Bahia». Rev. Museu Paul., 19: 1-326.

- $\quad 1938$ - "Catalogo das aves do Brasil e lista dos exemplares que as representam no Museu Paulista». 1.a parte. Rev. Museu Paul., 22: 1-556.

- $\quad$ - 1938a - «Nova contribuição à ornitologia amazonica. Estudo

- _ crítico de uma coleção de aves do Baixo Solimões e do Alto

- - Rio Negro». Rev. Museu Paul., 23: 493-604.

Plimmer H. G. - 1912 - On certain blood parasites». Jour. R. Micro. Soc., 133.

- $\quad-1912 \mathrm{a}$ - - On the blood parasites found in animals in the Zoological Gardens during the four years 1908-1911». Proc. Zool. Soc., 406-419.

- $\quad$ - 1912b - «Report on the deaths which occurred in the Zoological Gardens during 1911». Proc. Zool. Soc., 235-240.

- $\quad-1913$ - «Report on the deaths which occurred in the Zoological Gardens during 1912, together with a list of the bloodparasites found during the year". Proc. Zool. Soc., 141-149.

- $\quad-1914$ - - Report on the deaths which occurred in the Zoological Gardens during 1913, together with a list of the bloodparasites found during the year». Proc. Zool. Soc., 181-190. 
- $\quad-1915$ - - Report on the deaths which occurred in the Zoological Gardens during 1914, together with a list of the bloodparasites found during the year». Proc. Zool. Soc., 123-130.

- $\quad-1916$ - «Report on the deaths which occured in the Zoological Gardens during 1915, together which a list of the bloodparasites found during the year». Proc. Zool. Soc., 77-86.

- $\quad-1917$ - «Report on the deaths which occurred in the Zoological Gardens during 1916, together with a list of the bloodparasites found during the year». Proc. Zool. Soc., 27-35.

PRImıo, R. di - 1925 - «Contribuição para o estudo das Hemogregarinas Brasileiras». Rio. Tip. Leuzinger.

- $\quad$ - 1934 - «Sobre a presença do Haemoproteus columbae no - - Rio Grande do Suln. Arq. Riogrand. Med. (2) 3-4.

Ress, J. \& NobreaA P. - 1936 - Doenças das aves. (Tratado de Ornitopatologia). S. Paulo, Weiszflog Irmãos Inc.

Rosenbusch, F. - 1932 - «Toxoplasmosis avium en los canarios». 7a. Reun. Soc. Arg. Patol. Reg. Norte, 904-906.

Scort, H. H. - 1926 - -Report on the deaths occurring in the Society's Gardens during the year 1925». Proc. Zool. Soc., 231-244.

- $\quad-1927$ - «Report on the deaths occurring in the Society's Gardens during the year 1926». Proc. Zool. Soc., 173-198.

- $\quad-1928$ - «Report on the deaths occurring in the Society's Gardens during the year 1927». Proc. Zool. Soc., 81-119.

Splendore, A, - 1908 - «Un nuovo parasita de' conigli incontrato nelle lesioni anatomiche d'una malattia che ricorda in molti punto il Kalaazar dell'uomo». Nota preliminare Rev. Soc. Scient. S. Paulo, 3 (10 e 12) 109-112.

- $\quad 1909$ - Comunicação na sessão de 17 de Fevereiro de 1910, da Soc. Scient. de S. Paulo. Rev. Soc. Scient. S. Paulo, 4: $167-168$.

- $\quad-1909 \mathrm{a}-$ «Sopro un nuovo protozoo parasita de' conigli.. 2a. nota preliminare». Rev. Soc. Scient. S. Paulo, 4: 75-79.

- 1910 - «Trypanosoma di ucelletti e di pesci brasiliani». Rev. Soc. Scient. S. Paulo, 5: 6-13.

Vieira, C. C. - 1935 - «Os cotingideos do Brasil». Rev. Museu Paut., 19: 327-397.

- $\quad 1936$ - «Nomes vulgares de aves do Brasil». Rev. Museu Paul, 20: $437-490$.

WenYon, C. M. - 1926 - «Protozoology». New York, Bailliere, Tindall \& Cox. 